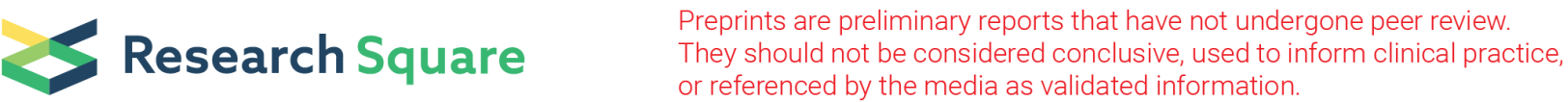

\section{Biomimetic nanomedicine-triggered in situ vaccination for innate and adaptive immunity activations for bacterial osteomyelitis treatment}

Han Lin

State Key Lab of High Performance Ceramics and Superfine Microstructure, Shanghai Institute of Ceramics, Chinese Academy of Sciences, Shanghai 200050, P. R. China.

\section{Chuang Yang}

Department of Orthopaedics, Shanghai Jiao Tong University Affiliated Sixth People's Hospital, Shanghai Jiao Tong University

\section{Min Ge}

State Key Laboratory of High Performance Ceramics and Superfine Microstructures, Shanghai Institute of Ceramics, Chinese Academy of Sciences

\section{Yao Luo}

Department of Orthopaedics, Shanghai Jiao Tong University Affiliated Sixth People's Hospital, Shanghai Jiao Tong University

\section{Xianlong Zhang}

Department of Orthopaedics, Shanghai Jiao Tong University Affiliated Sixth People's Hospital, Shanghai Jiao Tong University

Jianlin Shi ( $\nabla_{\text {jlshi@mail.sic.ac.cn ) }}$

State Key Laboratory of High Performance Ceramics and Superfine Microstructures, Shanghai Institute of Ceramics, Chinese Academy of Sciences https://orcid.org/0000-0001-8790-195X

\section{Article}

Keywords: biomimetic nanomedicine, in-situ vaccination, immune activation, osteomyelitis

Posted Date: July 13th, 2021

DOI: https://doi.org/10.21203/rs.3.rs-633609/v1

License: (c) (i) This work is licensed under a Creative Commons Attribution 4.0 International License. Read Full License

Version of Record: A version of this preprint was published at ACS Nano on March 22nd, 2022. See the published version at https://doi.org/10.1021/acsnano.1c11132. 


\section{Abstract}

The development of bacterial vaccines for inducing immunoresponse against infectious diseases such as osteomyelitis is of great significance and importance. However, the responsiveness of bacterial immunotherapy remains far from being satisfactory largely due to the erratic antigen epitopes of bacteria. Herein, we report an in situ vaccination strategy for the immunotherapy of bacterial infection based on an osteomyelitis model using a biomimetic nanomedicine named as HMMP, which was constructed by engineering PpIX-encapsulated hollow $\mathrm{MnO}_{\mathrm{x}}$ with a hybrid membrane exfoliated from both macrophage and tumor cell lines. The as-established HMMP features a burst bacterial antigen release as the in situ vaccine by the augmented sonodynamic treatment, and the resultant priming of antigen presenting cells for the following activations of both cellular and humoral adaptive immunities against bacterial infections. This treatment regimen not only triggers initial bacterial regression in established osteomyelitis model, also simultaneously generate robust systemic antibacterial immunity against poorly immunogenic secondary osteomyelitis in the contralateral knee as well, and additionally, confers long-lasting bacteria-specific immune memory responses to prevent infection relapse. Thus, our study provides a proof of concept of in situ vaccination for the activation of both innate and adaptive antibacterial immune responses, providing an individual-independent bacterial immunotherapy.

\section{Introduction}

Osteomyelitis is a kind of chronic infection diseases of bone with poor prognosis and low responsiveness to traditional antibiotic treatment owing to the unique anatomical and physiological characteristics of bone as well as the biofilm formation on implants ${ }^{1,2}$. The clinical standard-of-care treatments for osteomyelitis consist of surgical debridement and antibiotic chemotherapy, which, unfortunately, is able to temporally reduce the bacterial load, while $40 \%$ of the patients still suffer from recurrent and persistent infections. ${ }^{3}$ The alarming growth of the antibiotic-resistant superbugs is currently driving the advances of antibiotic-alternative strategies, such as photothermal and photodynamic nanomedicine. ${ }^{4,5}$ However, a crucial drawback of these methods is the limited depth of light penetration, making them impotent in eliminating deep bone infections. ${ }^{6}$ The emergence and circulation of multidrug-resistant strains, such as Staphylococcus aureus (S. aureus) and Mycobacterium tuberculosis (M. tuberculosis), are largely attributed to their capability to mount effective anti-immune responses ${ }^{7,8}$. Invading pathogens could inhibit antigen presentations by camouflaging their antigen epitopes to escape from immune surveillance, reprogramming macrophages to an M2 phenotype and/or even killing antigen-presenting cells. ${ }^{9,} 10$ In addition, the adaptive immunity would be deviated from homeostasis towards immune suppression by exhibiting signs of lymphocyte apoptosis ${ }^{10}$ and T cell exhaustion, ${ }^{11}$ causing persistent and recurrent infections, or even tissue death ${ }^{12}$ Nevertheless, with the rapid development of nanotechnology, rationally designed nanomedicine may play key roles in the immunotherapy against osteomyelitis from both bacterial and immunological viewpoints. 
Immunotherapy depends heavily on the immune responses which are largely affected or even countered by dysregulated pathogen's immune-microenvironment in, e.g., cancer ${ }^{13}$ and autoimmune diseases. ${ }^{14}$ In particular, cancer nanovaccines composed of tumor-specific antigens and adjuvants for actively inducing tumor-targeted immune responses, has been extensively studied and herald a breakthrough in the treatments of cancer metastases and recurrence ${ }^{15}$. Conventional nanovaccines necessitate exogenous antigens and adjuvants to work together to elicit antigen-specific immunity, while alternatively a recently explored strategy for cancer immune-therapeutics called 'in situ vaccination' directly utilizes autologous tumour-cell in vivo as antigens to stimulate comprehensive immune responses, ${ }^{16}$ in which nanoparticles possessing inherent immunogenic properties are utilized to activate the immune response and/or overcome local immunosuppression microenvironment of target tumours, and consequently potentiate systemic antitumour immunity for tumor immunotherapy. By sharp contrast, nanovaccines for osteomyelitis have been minimally explored as limited by the bacterial antigenic hypervariability 9,17 and microbiome heterogeneity of bacterial infection, ${ }^{18,19}$ not to mention 'in situ vaccination' using an immunostimulatory nanoplatform for reversing the immune-suppressive microenvironment of osteomyelitis to immune-infiltrated one, which remains a major challenge in the treatment of osteomyelitis.

Biomimetic nanotechnology offers promising alternatives for constructing disease-targeted nanoplatforms. ${ }^{20}$ Made by coating diverse cell membranes onto synthetic nanoparticulate cores, the asprepared membrane-engineered nanoplatforms inheriting antigenic profiles from source cell lines behavior as source cell lines possessing their complete membranous functions in their follow-up biomedical trials. ${ }^{21}$ For instance, macrophage membrane-encapsulated gold nanocages precisely is capable of navigating nanoparticles to infections position for a targeted photothermal therapy. ${ }^{22}$ Cancer cell membranes are used as a potent antigen source by the addition of a second adjuvant, leading to a robust immunostimulatory response and the multi-antigenic vaccacination. ${ }^{23}$ Furthermore, the hybrid cellular membrane nanovesicles inherently mimic the characteristics from two or more source cell lines, bestowing combined functions such as specific tumor targeting and tumor immunological microenvironment remodeling. ${ }^{24}$ To further augment the therapeutic effect of biomimetic nanotechnology, a paralleled integration between photothermal therapy (PTT) and chemotherapy also demonstrates synergetic outcomes of high-efficiency. ${ }^{21}$ Nevertheless, shell structured immunotherapy for osteomyelitis has not yet been reported to our best knowledge.

Here we present a hybrid nanovesicle consisting of functional core and cellular membrane shell for patient-specific sono-immunotherapy of osteomyelitis by an in situ bacterial vaccination strategy dependent on bacteria-associated antigens (BAA) and immune-activating tumor antigens. A hollow manganese oxide $\left(\mathrm{MnO}_{\mathrm{x}}\right)$ nanoparticle encapsulating a FDA-approved sonosensitizer protoporphyrin IX (PpIX) was first created as the supporting core, followed by co-extrusion with hybrid membrane vesicles integrating murine mammary carcinoma cell line 4T1 and murine macrophage cell line RAW264.7, to obtain the final biomimetic product of hybrid membrane@MnO ${ }_{x} @ P p I X$ (noted HMMP). Immunogenic 
sonodynamic therapy (SDT) for osteomyelitis has been achieved in synergy with source tumor cell membrane as immune adjuvant that recruit and activate antigen presenting cells (APCs) at the infection site where the bacterial associated antigens are released from the killed bacteria by SDT and antibacterial adaptive immune response is thus induced and further amplified (Scheme 1). This smart design elaborately bypasses the need of antigen delivery in traditional anti-infection vaccination, and triggers an in situ antibacterial vaccination in the following several ways: (i) HMMP nanoparticles recognize and enrich at the infectious sites with plenty of marks and receptors under the guidance of macrophage membranes. (ii) $\mathrm{MnO}_{x}$ decomposes under acidity or reacts with $\mathrm{H}_{2} \mathrm{O}_{2}$ to produce $\mathrm{O}_{2}$ in the infectious microenvironment (IME) facilitating the sustained ROS production via PpIX-based sonodynamic therapy, ${ }^{25,26}$ which would trigger bacterial death and antigen release. (iii) $\mathrm{Mn}^{2+}$ in combination with 4T1 tumor cell membrane antigens recruit dendritic cells (DC) and macrophage, and promote their maturations and antigen presentation, ${ }^{27}$ meanwhile, the anti-tumor adaptive immune cells show potent cross-reactivity with bacterial peptides. ${ }^{28}$ This HMMP biomimetic system exhibits strikingly high efficacy in inducing in situ vaccination on osteomyelitis model in mice, by efficiently activating both innate and adaptive immune responses against bacteria-derived acute osteomyelitis and its later recurrence. Notably, the HMMP nanoparticle triggered the systemic immune responses and conferred a complete, whole-stage protection against the bacteria-derived acute osteomyelitis challenge at the contralateral knee, and moreover, elicited long-lived B-cell and T-cell immunity to prevent recurrent infections. This work presents a general strategy for efficient in situ infection vaccination and prevention, featuring both the immunogenic death of bacteria and APC activation by the constructed biomedical nanoplatform.

\section{Results And Discussion}

\section{Preparation of HMMP and ROS generation by HMMP- mediated SDT}

The HMMP nanovaccines were prepared using an established film hydration method as shown in Fig. 1a. In this process, the porous hollow $\mathrm{MnO}_{\mathrm{x}}$ supporting core was synthesized according to a previously reported method ${ }^{25}$. Then, PpIX was loaded via magnetic rotary stirring route to yield $\mathrm{MnO}_{\mathrm{x}} @ \mathrm{PpIX}$. Hybrid membranes were formed by fusing murine mammary carcinoma cell line 4T1 (4T1 cells) and murine macrophage cell line RAW264.7 (RAW264.7 cells) together, which were then co-extruded with $\mathrm{MnO}_{\mathrm{x}} @ \mathrm{PpIX}$ (note MP) to obtain the final product (hybrid membrane@MnO @ @PpIX, HMMP). The UV - vis absorption spectra reveal the characteristic peak of PpIX at $407 \mathrm{~nm}$ for MP, and the loading efficiency was calculated to be $66.73 \%$ (Figure S1). Transmission electron microscopy (TEM) images verify the presence of a thin organic layer on the surface of HMMP, compared with the bare MP nanospheres, indicating the successful coating of hybrid membranes (Figure S2a-c). Uranyl acetate staining reveals that HMMP has a typical spherical core-shell structure, which is with a result of unilamellar membrane coating around the spherical supporting core (Fig. 1b). ${ }^{29}$ The uniform distributions of typical membrane elements such as C, $\mathrm{N}, \mathrm{Cl}, \mathrm{S}$ and $\mathrm{P}$ on the HMMP nanoparticles further validate the successful membrane incorporation onto 
the outer surface of MP cores (Fig. 1c and Figure S2d). Translocation of membrane protein contents onto the MP core was further examined via immunoblot analysis. SDS-page and Coomassie Brilliant Blue staining show that HMMP has retained the major membrane protein features of the sourcing cell lines (Fig. 1d). Western blot analysis further confirm that the HMMP contains the specific markers of individual cell membranes, including TLR6, a major bacterial receptor on macrophages, ${ }^{22}$ and E-cadherin, an important surface protein for $4 \mathrm{~T} 1$ breast tumor cell colonization and metastasis (Fig. 1e) ${ }^{30}$ The membrane cloaking resulted in an increase in the hydrodynamic diameter by $\sim 20 \mathrm{~nm}$ in comparison with the bare $\mathrm{MnO}_{\mathrm{x}}$. The surface zeta potential of $\mathrm{HMMP}$ is less negative than $\mathrm{MnO}_{\mathrm{x}}$, while close to that of hybrid membrane vesicles (Fig. 1f).

Biodegradation behavior of nanomaterials is a prerequisite for potential in vivo practices. The degradation process of $\mathrm{MnO}_{x}$ was investigated under the presence of glutathione $(\mathrm{GSH}, 4 \mathrm{mM})$, mimicking the $\mathrm{GSH}$-overexpressed bacterial biofilm microenvironment in vivo. $\mathrm{MnO}_{\mathrm{x}}$ shows a rapid degradation profile in the reductive condition, collapsing into fragments upon exposure to GSH for $24 \mathrm{~h}$ as evidenced by TEM imaging (Fig. 1g). To test whether the degradation of $\mathrm{MnO}_{\mathrm{x}}$ could induce the release of PpIX, MP was exposed to GSH as above. HMMP exhibited a rapid release of approximately $80 \%$ of the loaded PpIX in $24 \mathrm{~h}$ in the reductive microenvironment (Fig. 1h). To investigate the augmented SDT efficacy of HMMP, 1,3-diphenylisobenzofuran (DPBF) was applied to monitor the ultrasoundtriggered singlet oxygen $\left({ }^{1} \mathrm{O}_{2}\right)$ generation. The characteristic absorption peak intensity of DPBF at $420 \mathrm{~nm}$ showed significantly faster decrease after treated with HMMP than those treated with PpIX alone, indicating the substantial ${ }^{1} \mathrm{O}_{2}$ generation derived from PpIX-based sonodynamic treatment (Fig. 1i-j).

\section{HMMP-potentiated in situ burst bacterial antigen release}

Recognition of microbial pathogens using pattern recognition receptors such as toll-like receptors (TLRs), is essential for macrophages to initiate the antibacterial immune responses. In a recent study focusing on macrophage targeting, gold nanocages coated with macrophages membrane show enhanced bacterial targeting ability both in vitro and in vivo. ${ }^{22}$ We therefore employed in vivo bioluminescence imaging (IVIS) to track Cy5.5-loaded nanoparticles in mice osteomyelitis model after intravenous injection. Strong fluorescence was observed in vivo at the infected knee of mice treated with HMMP, while negligible fluorescence could be detected in the mice treated with bare MP. Ex vivo imaging of major organs reveals that the fluorescence signal is mainly located at the infected knee in HMMP group. By contrast, the fluorescence signal mainly distributed in the liver and kidney in control group (Fig. 2a). Semi-quantitative analysis further proves the high accumulating efficiency of HMMP at the infectious site (Figure S3). Better accumulation of HMMP at the infection site favors the SDT efficacy elevation and immune stimulation.

$\mathrm{MnO}_{\mathrm{x}}$ nanoparticles feature a marked catalytic activity for IME-enriched $\mathrm{H}_{2} \mathrm{O}_{2}$ decomposition into water and oxygen, ${ }^{25}$ which endows HMMP with oxygen production capacity. To verify this, HMMP was intravenously administrated on an infectious implant model in mice, and the subcutaneous oxygen level 
was monitored by photoacoustic (PA) imaging. In 60 min post-injection, a significant elevation of subcutaneous oxygen level was found in the infectious implant model rather than in the sterile implant model (Fig. 2b and Figure S4), verifying the potent oxygen producing capacity of HMMP in the IME in mice. The generated oxygen would fuel the ROS generation by SDT, resulting in HMMP-enabled and selfsustained SDT for bacteria and biofilm clearance.

Continuous low-dose stimulation under chronic infection will promote a phenotype of immune suppressive state by inducing $T$ cell exhaustion and derailing the acquisition of memory B cells ${ }^{31}$. Thus, the burst bacterial death may reverse the immune suppressive response and trigger long term antibacterial immunity. Our data suggest that HMMP has triggered massive reductions of in vitro bacterial cell viabilities on both planktonic and biofilm models of $S$. aureus and $E$. coli (Fig. 2c). Compared with the PpIX based SDT alone, MP was able to significantly enhance antibacterial efficacy owing to the augmented SDT. Besides, HMMP resulted in an enhanced bacterial killing efficacy compared with MP due to the active targeting effect of the cloaked RAW264.7 membrane components to the bacteria. Transmission electron images show the prominent disruption of HMMP-treated bacteria, featuring large defects in the cell wall and severe leakage of intrabacterial contents (Fig. 2d). We further investigated the membrane integrity using SYTO9 and propidium iodide (PI) staining. A clear loss of membrane integrity was observed in the bacteria treated with HMMP (Fig. 2e). Besides, HMMP triggered a significant loss of membrane potential as measured by $\mathrm{DiOC}_{2}$ staining (Fig. 2f). ${ }^{7}$ All the above results suggest that HMMP is capable of disrupting the integrity of bacteria and consequently promote the outward release of bacterial antigens. In the meantime, we analyzed the intrabacterial ROS generation performance to disclose the underlying antibacterial mechanism induced by HMMP. A significantly higher level of ROS was generated in the bacteria treated with HMMP than that in the other groups (Fig. $2 \mathrm{~g}$ and Figure S5). Such a SDT-triggered ROS generation for bacteria disruption is further augmented and sustained by the $\mathrm{MnO}_{\mathrm{x}}$-catalyzed $\mathrm{O}_{2}$ production and the macrophage membrane mediated specific targeting ${ }^{32}$. Thus, the highly efficient ROS production by HMMP-stimulated SDT triggers a burst bacterial antigen release, which will activate antigen presenting cells and induce substantial host immune responses (Fig. 2h). To detect the possible cytotoxicity of HMMP stimulation towards host cells, the HMMP nanoparticles were co-cultured with MC3T3-E1 cell line and exposed to US treatment as above mentioned. The CCK8 assay shows unaffected proliferation of MC3T3-E1 cells by HMMP-enabled SDT, indicating a safe and biocompatible potential of HMMP nanoparticles. (Figure S5).

\section{HMMP-enhanced bacterial phagocytosis and APCs activation}

Before employing HMMP as an emerging immune adjuvant in osteomyelitis treatment, we first tried to verify its inherent immunogenicity in priming APCs including macrophages and DCs. Activated macrophages (M1 phenotype) have been supposed to play a major role in defending against foreign pathogens, but the cues such as bacterial polyphosphates from the immunosuppressive chronic IME would alternatively guide the macrophages to polarize toward M2 phenotype featuring bacterial 
phagocytosis and antigen presentation. ${ }^{33}$ To evaluate the potential of HMMP in determining macrophage polarization, the phagocytosis capacity and macrophage phenotype markers were analyzed.

Macrophages treated with HMMP show marked increase in the phagocytosis rate toward both $S$. aureus and E. coli in comparison to the control group (Fig. 3a and Figure S6). Meanwhile, flow cytometry results demonstrate that HMMP is able to potentiate a shift in macrophage polarization toward M1 phenotypes, as evidenced by a significant increase in CD80 expression and decrease in CD206 expression (Fig. 3b, Figure S7 and S9a). ${ }^{34}$ Dendritic cells, as another important type of antigen-presenting cells in infectious diseases, are highly effective in initiating and regulating both innate and adaptive immunities. ${ }^{35} \mathrm{CD} 80$ and CD86 are the major co-stimulatory molecules expressed on DCs, the level of which reflects the DCs maturation status. Bone marrow-derived dendritic cells (BMDCs) cultured with HMMP show a significantly increased proportion of $\mathrm{CD} 80^{+} \mathrm{CD} 86^{+}$cells (Fig. 3c and Figure S8, S9b), which are positively correlated to the antigen presenting capacity. The phenotype changes of the above two typical APCs prompted us to investigate the genomic changes caused by HMMP.

To map the genomic landscape of APCs affected by HMMP, we conducted RNA-seq analysis for Raw264.7 macrophages. Over 2900 differentially expressed genes (DEGs) associated with HMMP intervention were identified, which were dominated by genes associated with proinflammatory responses (Fig. 3d). Kyoto Encyclopedia of Genes and Genomes (KEGG) enrichment analysis reveals the enriched DEGs after HMMP treatment in antigen processing and presentation-associated signaling pathways, such as NOD-like receptor, Toll-like receptor and RIG--like receptor signaling pathways (Fig. 3e and Figure S10). Gene signature analysis indicates that the key genes in regulating antigen processing and presentation such as Tap1 and Tnf have been significantly upregulated (Fig. 3f), which would amplify the antibacterial activity of the innate immunity and facilitate the activation of adaptive immunity. ${ }^{36}$ In addition to the enhanced DCs' maturation, the HMMP treatment led to significant upregulations of proinflammatory cytokine genes such as IL6, IL12, IL1 $\beta$ and TNF- $a$, and the downregulation of antiinflammatory cytokine genes including IL10 and TGF- $\beta$ (Fig. $3 g$ and Figure S11). The changes in the transcriptional profile of cytokines further support the findings of immune-cell activation and antibacterial inflammation. To conclude, the above studies prove that HMMP is a powerful immune adjuvant for activating APCs such as macrophages and DCs, proving its potential as an effective nanovaccine (Fig. 3h).

\section{HMMP-induced osteomyelitis regression and antibacterial immune responses}

Given the observed in vitro results of HMMP functions on activating bacterial phagocytosis and APCsmediated antigen presentation, we speculated that HMMP would be able to inflame the immunesuppressive implant-associated IME and generate in situ vaccination on in vivo osteomyelitis model. To test this hypothesis, we first evaluated the knee perimeter, microbiological changes and radiologic features of mice burdened with $S$. aureus osteomyelitis (Fig. 4a). Following osteomyelitis induction, mice in the control group developed sever swelling and purulent knee joints, whereas mice treated with HMMP induced significantly smaller knee perimeter without significant infection signs (Fig. 4b-c). Colony-count 
assay using the harvested bone tissues and implants shows a marked reduction of bacterial colonies in the HMMP group (Fig. 4d). As can be observed from the MRI imaging, a significantly decreased T2 signal in the HMMP group indicates a largely alleviated infection severity (Fig. 4e). Similarly, Gimesa staining shows a massive bacteria infiltration in methicillin-resistant Staphylococcus aureus (MRSA)-infected bone, in contrast, well-structured tissues can be observed in HMMP group (Fig. 4f), suggesting a thorough bacterial eradication.

Encouraged by these results, we performed RNA-seq analysis to highlight the pathway signatures in driving antibacterial responses in vivo. Over 2200 DEGs (Fig. 4g) were identified in the bone marrow of mice treated with HMMP compared to the control group. Positively correlated signatures between HMMP intervention and immune response-associated signaling pathways are the strongest among the experimental groups (Fig. 4h-i and Figure S12-13). Specifically, signaling pathways related with bacterial recognition and presentation, such as toll-like receptor, nod-like receptor and C-type lectin, are all upregulated in the HMMP group. Significantly, innate immune pathways responsible for bacterial clearance such as leukocyte transendothelial migration and NK cell-mediated cytotoxicity are upregulated in HMMP group. Pathway signature analysis also reveals that the adaptive immune system is highly active in HMMP group, with high levels of B cell and T cell signaling, as well as differentiation of T helper cells compared to the other groups. Further gene signature analysis show that key genes involved in antiinfection immune responses such as Mapk14 and Cdc42, are significantly upregulated in the bone of mice treated with HMMP. Moreover, these transcriptomic profiles also suggest that HMMP treatment can inflame the IME and activate the antibacterial immune responses.

\section{HMMP-stimulated immunogenic infection microenvironment and in situ vaccination}

To further elucidate the underlying mechanism of the alterations in the transcriptomic profiles, we mapped the cellular composition in the bone marrow. First, to visualize the immune microenvironment in the infected bone marrow grossly, $\mathrm{CD} 45^{+}$immune cells were stained. Mice treated with HMMP show a significantly enhanced infiltration of $\mathrm{CD} 45^{+}$immune cells, indicating an inflamed immune response (Fig. 5a). Infection-associated macrophages (IAMs) possessing M1 phenotypes are responsible for bacterial clearance through phagocytosis and antigen dependent facets ${ }^{33}$. Thus, we sought to determine the phonotype of IAMs for HMMP-carrying mice. We found that IAMs of HMMP-treated mice were indeed polarized toward M1 phenotypes, as proved by an enhanced expression of iNOS and reduced expression of CD206 in the immunofluorescence staining (Fig. 5b).

Looking specifically into the immune cell composition, we found that HMMP treatment led to the strongest infiltration of key drivers of antibacterial immune responses as determined by detailed flow cytometry analysis (Fig. 5c-d and Figure S14-22). A significant amount increase of M1 macrophages $\left(\mathrm{CD} 80^{+} \mathrm{CD} 11 \mathrm{~b}^{+} \mathrm{F} 4 / 80^{+}\right)$and decrease of $\mathrm{M} 2$ macrophages $\left(\mathrm{CD} 206^{+} \mathrm{CD} 11 \mathrm{~b}^{+} \mathrm{F} 4 / 80^{+}\right)$were elicited by HMMP in the IME. Besides, the percentage of mature DCs $\left(\mathrm{CD} 11 \mathrm{C}^{+} \mathrm{MHC}-\mathrm{II}^{+} \mathrm{CD} 80^{+} \mathrm{CD} 86^{+}\right)$increased sharply in the bone marrow of HMMP-treated mice. A higher population of mature DCs as a kind of cancer vaccine, has been reported to induce a stronger adaptive immunity against cancer, ${ }^{37}$ by analogy, 
we hypothesize that the elevated mature DCs fraction as a kind of bacteria vaccine would result in adaptive immunity sensitization with enhanced bacterial killing capacity. As expected, enhanced T cell infiltrations, upon both $\mathrm{CD} 4^{+} \mathrm{T}$ helper cells $\left(\mathrm{CD} 45^{+} \mathrm{CD} 3^{+} \mathrm{CD} 4^{+}\right)$and $\mathrm{CD} 8^{+}$cytotoxic $\mathrm{T}$ lymphocytes $\left(\mathrm{CD} 45^{+} \mathrm{CD} 3^{+} \mathrm{CD} 8^{+}\right)$, were observed in the HMMP-treated mice. It has been known that chronic infection, especially the hypoxic biofilm formation in IME drives T cell exhaustion, resulting in a hypofunctional fate of much compromised bacterial clearance capacity. Thus, the increased infiltrations of both $\mathrm{CD} 4^{+}$and $\mathrm{CD}^{+} \mathrm{T}$ cells are vitally important for the infected host to summon an effective antibacterial response.

In addition, to elicit protective humoral immunity, B cells need to differentiate into either long-lived plasma cells that produce antibody, or memory B cells that respond to the re-exposure of pathogens. ${ }^{38}$ Our outcomes show that HMMP can generate a nearly 2.0-fold increase in the fraction of plasma cells $\left(\mathrm{CD} 45^{+} \mathrm{CD} 19^{+} \mathrm{CD} 138^{+}\right)$in the infected bone, compared to the untreated group. Such an enhanced production of long-lived plasma cells provides a lifelong source of protective antibodies against invading pathogens which is essential for vaccination ${ }^{39}$. Hiding inside of host cells is a tricky strategy of immune evasion adopted by the invading microbes. NK cells and CD8 $+\mathrm{T}$ cells are respectively the major innate and adaptive lymphocytes to find and kill the infected cells. Notably, HMMP administration led to the highest recruitment of NK cells $\left(\mathrm{CD} 45^{+} \mathrm{NK} 1.1^{+}\right)$to the infected bone marrow. The strongly promoted infiltrations of both NK cells and CD8 + T cells by HMMP would result in effective immune clearance of intracellular bacteria.

Furthermore, one hallmark of immunosuppressive landscape is the massive infiltrations of regulatory $T$ $\left(T_{\text {reg }}\right)$ cells and myeloid-derived suppressor cells (MDSCs) in the bone marrow. To quantify the effect of HMMP on modulating the immunosuppressive IME, the infiltrations of $\mathrm{T}_{\text {reg }}$ cells and MDSCs were also analyzed. As expected, mice treated with HMMP exhibit a significant decrease in the $T_{\text {reg }}$ fraction of $C D 4^{+}$ cells $\left(\mathrm{CD}^{+} \mathrm{CD} 4^{+} \mathrm{FoxP}^{+}\right)$, indicating a much-weakened immunosuppression. Similarly, the proportions of both $\mathrm{CD} 45^{+} \mathrm{Gr} 1^{\text {high }} \mathrm{CD} 11 \mathrm{~b}^{+}$granulocytic MDSCs and $\mathrm{CD} 45^{+} \mathrm{Gr} 1^{\text {int }} \mathrm{CD} 11 \mathrm{~b}^{+}$monocytic MDSCs decreased significantly in the HMMP-treated mice. As the massive infiltrations of $\mathrm{T}_{\text {reg }}$ cells and MDSCs would disenable $T$ cells and block immune cell effector functions ${ }^{40}$, thus, the HMMP-induced $\mathrm{T}_{\text {reg }}$ cell and MDSCs depletions are proposed to be an effective pathway to enhance the efficacy of bacterial immunotherapies.

Encouraged by the above results, we further tested the levels of cytokines and antibodies in IME in the infected bone to highlight their contributions in driving antibacterial immunity. The mice treated with HMMP show marked amount increases of pro-inflammatory cytokines such as IFNY, TNFa and IL12 and decrease of anti-inflammatory cytokines such as IL10 (Fig. 5e), further evidencing that HMMP can elicit robust antibacterial immune responses in a poor immunogenic osteomyelitis. The induction of protective antibody responses is an essential part in the development of vaccination against infectious diseases. The administration of HMMP as a composite antigen induced strong IgM and IgG antibody responses featuring $\sim 2$-fold post enhancements of both IgM and IgG titers (Fig. 5f), indicating the effective 
induction of B cell-mediated humoral immunity upon HMMP vaccination. In conclusion, HMMP can indeed inflame both innate and adaptive antibacterial immune responses: the former is simply by macrophage M1 polarization and activation, while the latter is achieved by both APCs-activated T cell immunity under the mediation of HMMP-assisted SDT, and HMMP-initiated and protective antibodyenabled humoral immunity by $\mathrm{B}$ cells. These immuno-responses evidence successful in situ vaccination against bacterial infections (Fig. $5 \mathrm{~g}$ ).

\section{HMMP-inflamed transferable antibacterial immune responses}

Uncontrolled local bacterial infections can progressively develop into fatal status characteristic of bacteremia and distant organ injuries. ${ }^{6,7}$ Besides, infection-associated immunosuppression would predispose the host to secondary infections ${ }^{41}$. To assess whether the in situ nanovaccination could induce potent systemic antibacterial immune responses which would prevent the formation of distant infection foci, we constructed a contralateral osteomyelitis model after the immunization by HMMP administration (Fig. 6a). Briefly, an in situ osteomyelitis model was firstly built, and then mice were immunized with HMMP. Afterwards, another osteomyelitis model was established in the contralateral knee. In 14 days after the model establishment, bacterial analyses of the contralateral infected knee including perimeter measurements, CFU counts, MRI evaluation and Gimesa staining were conducted. Besides, the spleens of mice were harvested for immunological analysis. Mice immunized with HMMP show sharply reduced infection severity as evidenced by the largely alleviated knee joint swelling (Fig. 6bc). CFU counts reveal a much-decreased bacterial burden in mice immunized with HMMP (Fig. 6d). Diffused edema and liquefactive necrosis in the joint cavity and surrounding muscles can be seen in the mice of control group in the MRI imaging, while the complete clearance of infectious lesions can be observed in mice treated with HMMP (Fig. 6e). Gimesa staining of pathological section of mice in the control group exhibit numbers of bacterial infiltration, whereas the tissues in the HMMP group show normal morphological features (Fig. 6f). Above results suggest that HMMP in situ vaccination could largely diminish the infection severity in the contralateral osteomyelitis model. To understand this, we evaluated the immune responses in the spleen, a major lymphoid organ specialized in systemic host defense against invading pathogens. Following antigen capture, abundant APCs migrate to the spleen and interact with the immune cells readily to elicit systemic antibacterial responses. Spleens of mice bearing osteomyelitis reveal potent immune responses, featuring antigen presentation and adaptive immunity including both cellular and humoral immunities (Fig. 6g-h and Figure S23-29).

Splenocytes collected from mice immunized with HMMP exhibit the highest infiltration of plasma cells, $\mathrm{CD}^{+}$and $\mathrm{CD} 8^{+} \mathrm{T}$ cells, as well as NK cells. The increased percentage of plasma cells among spleen $\mathrm{B}$ cells potentiate a high enough number of antibodies for specifically targeting the invading pathogens, indicating an effective humoral immunity. $\mathrm{CD} 8^{+} \mathrm{T}$ cell expansion in the lymphoid organ is also a major feature of systemic immunizations, which represents enhanced cellular immunity. HMMP inhibited the influx of regulatory immune cells including $T_{\text {reg }}$ cells and MDSCs, thus reduced the immunosuppressive potential of bacterial infection. Together, the immune cell profiles verify that HMMP induced both potent 
systemic humoral and cellular immunity against bacterial dissemination. To characterize the host immune response more completely, we performed ELISA analysis of sera cytokines and antibody levels (Fig. 6i). A significant amount increase in proinflammatory cytokines and decrease in anti-inflammatory cytokines are observed in the blood of mice treated with HMMP, suggesting that HMMP has initiated a proinflammatory cytokine storm for the amplifications of antibacterial immune responses and therapeutic effect. Besides, IgM and IgG responses in mice immunized with HMMP were markedly stronger than those in other groups, indicating a robust humoral immune response.

\section{HMMP-conferred long-lasting protective immunity against bacterial infections}

To explore the capability of HMMP nanovaccine to evoke anti-bacterial immune recall responses in a prophylactic setting, we next studied whether the HMMP could prevent infection recurrence after primary immune de-infection or not. To mimic a clinically relevant situation, osteomyelitis established in mice were first debrided by HMMP-induced immunotherapy, then the mice were re-challenged with $S$. aureus six weeks later (Fig. 7a). Notably, mice pre-immunized with HMMP has withstood the bacterial re-challenge, as evidenced by the slight infectious signs and bacterial count (Fig. 7b-f). The antigenic hypervariability ${ }^{9}$ 17 and microbiome heterogeneity ${ }^{18,19}$ of the bacterial infection impose the current bacterial vaccines with constricted breadth of bacteria-specific immune memory responses by both B cell and T cell. Thus, we sought to evaluate whether HMMP could elicit effective immune recall response or not by using flow cytometry analysis (Fig. 7g-i and Figure S30-37). As expected, substantially larger pools of memory B cells (B220 ${ }^{+} \mathrm{gG}^{+} \mathrm{IgD}{ }^{\text {low }}$ ) were elicited both in the bone marrow and in the spleen by HMMP than in the control group. To test the long-term antibody producing potential of memory B cells, we analyzed the IgG level in the bone marrow and spleen after bacterial antigen re-challenge (Fig. 7j). The IgG levels were significantly higher in mice pre-treated with HMMP than in the others, suggesting that memory B cells were predisposed to differentiate into plasma cells in HMMP pre-treated mice in the secondary response. ${ }^{31}$ Overall, HMMP immunization has induced affinity maturation of B cells to develop into memory B cells possessing antibody production and bacterial clearance capacity.

Analysis of memory T cells gives similar findings, such a HMMP-induced in situ vaccination triggered marked memory $T$ cell responses both in the spleen and bone marrow. Mice pre-treated with HMMP show a sharp increase in the $\mathrm{CD} 4^{+}$memory $\mathrm{T}$ cells $\left(\mathrm{CD} 4^{+} \mathrm{CD} 44^{\text {high }} \mathrm{CD} 62 \mathrm{~L}^{\text {low }}\right)$ and $\mathrm{CD} 8^{+}$memory $\mathrm{T}$ cells $\left(\mathrm{CD} 8^{+} \mathrm{CD} 44^{\text {high }} \mathrm{CD} 62 \mathrm{~L}^{\text {low }}\right)$ compared with those in the control group. These results once again highlight the effectiveness of HMMP nanovaccines in inducing durable immunological memory against infection relapse.

\section{Discussions And Conclusion}

Infection-induced immune cell apoptosis causes dramatic depletion of immune cells, including dendritic cells, B cells, NK cells, CD $4^{+}$and CD $8^{+}$T cells. ${ }^{12,42}$ The secondary clearance of apoptotic immune cells by DCs, macrophages and monocytes further triggers extensive immunosuppressive cytokine production 
and immune tolerance, largely deteriorating the host defense against pathogens. ${ }^{43}$ Besides, the expansion of regulatory $T$ ( $T_{\text {reg }}$ ) cell and myeloid-derived suppressor cell (MDSC) populations in sepsis contributes to the formation of a more immunosuppressive phenotype. ${ }^{12,42}$ Therefore, it is of great importance to restore the epigenetic, transcriptional and functional programs of immune responses during infection-induced tolerance.

To continuously adapt themselves for survival in osteomyelitis, microbes try to escape from the recognition and clearance by reducing the metabolism rate and frequently mutating their antigenic epitope. Concurrently, host immune factors tend to evolve themselves for adaption to an immunosuppressive state. Thus, a major hurdle in designing bacterial vaccines is to induce highly mutated antibodies onto the non-immunodominant epitopes in the IME. ${ }^{44}$ Conventional vaccines deliver one or several antigens and adjuvants to APCs for the elicitations of antigen-specific cytotoxic $T$ lymphocyte (CTL) and humoral responses, but their applications in anti-bacterial infections especially in the well-established ones have mostly failed due to the highly mutating antigenic epitope.

To expand the spectrum of antigenic epitopes in the vaccine, whole tumour cell individuals have recently been adopted as antigen sources for cancer vaccination ${ }^{45,46}$. But this approach involves complex extraction and purification of tumor cells from patients, and deactivation of the tumor cells in vitro, so that they can be reintroduced into the patients. The strategy of "in situ" vaccination provides an alternative but attractive approach that overcomes the challenges in defining specific antigens for vaccine development and in whole-cell vaccination preparation ${ }^{47}$, which outperforms most of previously reported immunotherapies in treating cancers ${ }^{46}$. This approach features the local immunosuppressive microenvironment regulation by directly administrating immunostimulatory reagents into the foci ${ }^{48}$, thus demonstrating great potentials in treating poor-immunogenic diseases including cancer and chronic infections.

Immunostimulatory reagent of potent APCs activation capacity is the key in engineering in situ vaccination. Tumor cells have been widely reported as antigens in cancer vaccination to initiate antitumor immune responses thanks to its well understood immunogenicity. In the current study, we explore, for the first time, the immunogenic properties of tumor cell membranes to endow the bacteria-infected host with robust antigen priming capacity, thus initializing anti-bacterial immune responses. Besides, $\mathrm{Mn}^{2+}$, the degradation product of the supporting core which can reshape the immune microenvironment ${ }^{27}$, acts in synergy with the tumor cell membrane to initiate the antibacterial immune responses. The phenotype polarization and transcriptional changes of macrophages and BMDCs in vitro driven by HMMP evidence the immunostimulatory effect of the designed nanoplatform.

Limited availability of infection-associated antigens (IAAs) would also reduce the clinical efficacy of infection vaccines. Continuous low-dose stimulation under chronic infection may induce an immunosuppressive state of $T$ cell exhaustion and incompetent $B$ cell responses. In the initial treatment of osteomyelitis, we adopted US-triggered SDT using PpIX-loaded nanomedicine to kill the bacteria and 
induce burst IAAs release for APC activation. SDT is a promising noninvasive therapeutic modality for the treatment of deep infectious lesions via the generation of ROS for IAA burst release, while $\mathrm{MnO}_{\mathrm{x}^{-}}$ catalyzed $\mathrm{O}_{2}$ production in the IME further augment the generation of intrabacterial ROS. Resultant IAAs of a wide spectrum of bacterial antigenic profiles, together with the concentrated and activated APCs, would trigger effective and specific bacteria and/or host in situ vaccinations.

Current clinical treatment of osteomyelitis typically includes debridement surgeries, two-stage revisions and long-term antibiotic suppression to prevent recurrence. The present HMMP vaccine can be singledose and intravenously injected to elicit robust humoral and cellular antibacterial immunity, and further evoke long-term memory immune responses. This treatment approach offers an efficient antibacterial effect against osteomyelitis in both local and systemic therapeutic settings, and subsequently provides a long-term protection from infection recurrence in the prophylactic settings. Also, the present "in situ" vaccination strategy could be combined with surgeries or antibiotics for enhanced anti-infection efficacy. Specifically, surgery is hard to debride all bacteria at the infectious site even with the implant being removed, which actually become a source for infection recurrence. Besides, in the cases of bacteremia or distant metastatic infection foci, patients are at high risk of infection recurrence. HMMP in situ vaccination is capable of initiating effective antibacterial immune responses against bacteria both locally and systematically by disrupting remaining or recurrent bacteria from the surgical debridement. Of note, this immunization regime is also applicable to osteomyelitis patients who cannot withstand the trauma of surgeries.

To conclude, we have here proposed a general strategy of in situ vaccination for the highly effective bacterial infection treatment, which features both the innate immunogenic bacteria disruption and adaptive immune-response activation by the antigen burst release-stimulated APC presentation during HMMP-enabled SDT. Both the cellular and humoral immunities have been verified in the adaptive immunity, together with the memory adaptive immunity preventing the recurrence of infections by the biomimetic nanoplatform. As the $\mathrm{MnO}_{\mathrm{x}}$ core, macrophage and 4T1 membranes are degradable, and PpIX is a FDA-approved sonosensitizers, the established HMMP nanomedicine features a promising clinical translation potential. This protocol enables the development of versatile in situ bacteria vaccinations against bacterial infection bypassing the needs of identifying individual IAAs and personalized manufacturing of vaccines.

\section{Methods}

Materials. 4T1 cells, RAW264.7 cells and MC3T3-E1 cells were obtained from Shanghai Institute of Cell Biology, Chinese Academy of Science. S. aureus (ATCC43300) and E.coli (ATCC35218) were purchased from the American Type Culture Collection. BMDCs were isolated from the bone marrow of 6-week-old C57 mice according to a precious reported method ${ }^{49}$. Male male BALB/c mice (6-8 weeks) and C57BL/6 mice (6-8 weeks) were purchased from Laboratory Animal Management Department, Shanghai Family Planning Research Institute. All of the animal experiments performed in this research were approved by the Institutional Animal Care and Use Committee of the Shanghai Sixth People's Hospital. 
Dulbecco's modified Eagle's medium (DMEM), 1640 media, penicillin and streptomycin, fetal bovine serum (FBS), PBS, SYTOX/PI staining kits and ACK lysis buffer were obtained from Thermo Fisher Scientific. Antibodies, True-Nuclear ${ }^{\mathrm{TM}}$ Transcription Factor Buffer Set, Cyto-Fast ${ }^{\mathrm{TM}}$ Fix/Perm Buffer Set used for flow cytometry were purchased from Biolegend company, if not specially indicated. TNFa, IL6 Elisa kits were purchased from R\&D Systems, IFNY and IL10 Elisa Kits were purchased from Dakewei Biotech. IgG and IgM Elisa kits were purchased from Crystal Chem. The titanium implant (plateau, $2 \mathrm{~mm} \times 1.5 \mathrm{~mm}$; stem diameter, $0.6 \mathrm{~mm}$; stem length, $2 \mathrm{~mm}$ ) used for osteomyelitis model was custom-made by Shanghai Sunshine-laser Company. Flow cytometric analysis was performed on FACSVerse flow cytometer (BD Biosciences). All experiments involving US treatment were performed with a US transducer (Chattanooga Co., USA).

Synthesis of HMMP. Hollow porous $\mathrm{MnO}_{\mathrm{x}}$ was synthesized with a previous reported method ${ }^{25}$. Briefly, $\mathrm{MnO}_{\mathrm{x}} @ \mathrm{SiO}_{2}$ was synthesized by simply mixing the as-made silica nanoparticles with $\mathrm{KMnO}_{4}$, utilizing the reduction reaction between organosilica and $\mathrm{KMnO}_{4}$. Then the silica template was removed with $\mathrm{Na}_{2} \mathrm{CO}_{3}$ solution, the obtained product was then washed with water thrice, yielding hollow porous $\mathrm{MnO}_{\mathrm{x}} \cdot \mathrm{MnO}_{\mathrm{x}}$ was mixed with PpIX in a ratio of 1:10, followed by magnetic rotary stirring for $12 \mathrm{~h}$ at room temperature. After washing with ethanol thrice, the product was then dispersed into water.

To extract membranes from Raw264.7 and 4T1 cells, the respective cells were suspended in hypotonic lysing buffer, followed by disrupting with a Dounce homogenizer. The homogenized solution was centrifuged at $20,000 \mathrm{~g}$ at $4^{\circ} \mathrm{C}$ for $30 \mathrm{~min}$. The supernatant was harvested and centrifuged at $100,000 \mathrm{~g}$ at $4^{\circ} \mathrm{C}$ for $35 \mathrm{~min}$. The pellets were harvested and washed with PBS containing protease inhibitor, and sonicated for $5 \mathrm{~min}$. The membrane vesicles were then obtained after extrusion through $400 \mathrm{~nm}, 200 \mathrm{~nm}$, and $100 \mathrm{~nm}$ polycarbonate porous membranes (Avanti Polar Lipids). The membrane vesicles from Raw264.7 and 4T1 cells were mixed in a weight ratio of 1:1, sonicated for $5 \mathrm{~min}$, followed by extrusion through 100-nm polycarbonate porous membranes. For membrane coating, the hybrid membrane was mixed with MP core at a weight ratio of 2:1, then sonicated for 2 min, followed by serially extrusion through $400 \mathrm{~nm}$ and $200 \mathrm{~nm}$ polycarbonate porous membranes.

Characterization. TEM, HR-TEM and elemental mapping was conducted on a JEM-2100F electron microscope operated at $200 \mathrm{kV}$. UV - vis - NIR absorption spectra were performed on a UV-3101PC Shimadzu spectroscope. Zeta potential measurements were performed on a Zetasizer Nanoseries (Nano ZS90, Malvern Instrument Ltd.). The diameters of different nanoparticles were analyzed with Nano Measure software. The degradation behaviors of $\mathrm{MnO}_{\mathrm{x}}$ were studied in redox conditions with $4 \mathrm{mM} \mathrm{GSH}$ under constant shaking. The samples were harvested at predetermined time (0, 3, 6, 12 and $24 \mathrm{~h})$, and observed with TEM. To study the PpIX release, a solution of MP was dialyzed against PBS with or without $4 \mathrm{mM}$ GSH under constant shaking, the amounts of PpIX release were measured at preset time using UVvis spectra.

RAW264.7 membrane vesicles, 4T1 membrane vesicles and HMMP containing equivalent total proteins were prepared using BCA assay (Beyotime). The proteins were resolved on SDS-polyacrylamide gel 
electrophoresis (SDS-PAGE) gels, followed by Coomassie Brilliant Blue staining or standard WB. The primary antibodies used were anti-TLR6 (Proteintech, 22240-1-AP) and anti-E-cadherin (Proteintech, 20874-1-AP). Blots were incubated with anti-Rabbit IgG, HRP-linked Antibody (Proteintech, SA00001-2). The signals were detected by the HRP-based chemiluminescence analysis on ChemiDoc (BioRad). To evaluate ROS generation, $20 \mu \mathrm{L}$ DPBF $\left(1 \mathrm{mg} \mathrm{mL}^{-1}\right)$ was added in $1 \mathrm{~mL} \mathrm{HMMP}\left(20 \mu \mathrm{g} \mathrm{mL}^{-1}\right)$, the mixture was then exposed to US stimulation $\left(40 \mathrm{kHz}, 1.5 \mathrm{~W} \mathrm{~cm}^{-2}\right)$. The absorbance changes of DPBF at $420 \mathrm{~nm}$ were detected and recorded. PBS and PpIX solution $\left(20 \mu \mathrm{g} \mathrm{mL}^{-1}\right)$ were used as controls.

Study design. For in vitro studies, cells or bacteria treated with PBS, PpIX, $\mathrm{MnO}_{\mathrm{x}} @ P$ PIX and hybridized membrane@MnO $@$ @PpIX were termed as Control, P, MP and HMMP, respectively. The naming method in vivo is in analogous to that in vitro. To load $\mathrm{PpIX}, \mathrm{MnO}_{\mathrm{X}}$ was mixed with $\mathrm{PpIX}$ in a ratio of 1:10 under ultrasonication, follow by magnetic rotary stirring, thus yielding $\mathrm{MnO}_{\mathrm{x}} @ \mathrm{PpIX}$.

Biosafety test. MC3T3-E1 cells ( $100 \mu \mathrm{L}, 5,000$ cells per well) were cultured in 96-well microplates overnight. Then, the culture media were replaced with $100 \mu \mathrm{L}$ of fresh media containing PBS or HMMP with gradient concentrations. After further culture for $24 \mathrm{~h}$, the cells were washed with PBS thrice, then CCK-8 solution was added. 90 min after the co-incubation, the absorbance at a wavelength of $450 \mathrm{~nm}$ was determined with a microplate reader (Bio-TekELx800, USA).

In vivo biodistribution of HMMP. Mice osteomyelitis model was first constructed. The method to establish osteomyelitis model was introduced in detail in the following "In situ" osteomyelitis model establishment. HMMP nanoparticles labelled with Cy5.5 $\left(100 \mu \mathrm{L}, 2 \mathrm{mg} \cdot \mathrm{mL}^{-1}\right)$ were intravenously injected 3 days after the induction of infection. MP nanoparticles labelled with Cy5.5 were used as control. Signals of HMMP nanoparticles were monitored with an IVIS fluorescence imaging system (VISQUE InVivo Elite) over time. $12 \mathrm{~h}$ after the injection, the major organs were harvested for IVIS observation.

In vivo detection of oxygen saturation by PA. Subcutaneous implant-related infection model and sterile model were constructed as follows. Six-week-old BALB/c mice were anesthetized with pentobarbital via intraperitoneal injection. After anesthesia, the back was shaved and disinfected, an incision was then made to expose the subcutaneous cavity. Next, a sterile polyethylene gasket (diameter: $6 \mathrm{~mm}$, thickness: $1 \mathrm{~mm}$ ) was placed into the cavity followed by wound suturing. Finally, $100 \mu \mathrm{L}$ of $10^{6} \mathrm{CFU} \mathrm{mL}^{-1} \mathrm{~S}$. aureus or PBS was injected onto the polyethylene gasket to establish infectious or sterile implant model, respectively. Three days after the surgery, mice were injected intravenously with HMMP $(100 \mu \mathrm{L}, 2 \mathrm{mg}$ $\left.\mathrm{mL}^{-1}\right)$. The oxygen levels in the subcutaneous region were monitored with PA imaging (Fuji).

In vitro antibacterial tests. $1 \mathrm{~mL}$ of $S$. aureus and $E$. coli at the concentration of $10^{6} \mathrm{CFU} \mathrm{mL}^{-1}$ were coincubated with PBS, P, MP or HMMP $\left(20 \mu \mathrm{g} \mathrm{mL}^{-1}\right)$ for $8 \mathrm{~h}$, followed by US irradiation $\left(40 \mathrm{kHz}, 1.5 \mathrm{~W} \mathrm{~cm}^{-2}\right.$, 1 min per cycle, 5 cycles). The bacterial suspension and adhered biofilm in the well were harvested, respectively. Bacterial viability from each sample was tested with CFU assay. The bacterial membrane integrity was tested with Live/Dead assay. Briefly, the bacterial suspension was spun down and wash with PBS for three times before the SYT09/PI staining was added. After co-incubation for 15 min in the 
dark, the solution was further washed with PBS for three times. An aliquot was aspirated and tested with flow cytometry. The procedure for the detection of membrane potential resembled the SYT09/PI staining protocol with the following exceptions. Bacterial samples were stained with $3 \mathrm{mM} 3,3^{\prime}-$

diethyloxacarbocyanine $\left(\mathrm{DiOC}_{2}\right)$ dye. The $\mathrm{DiOC}_{2}$ dye forms tetramers that fluoresce at $630 \mathrm{~nm}$ when the membrane potential is intact, while it forms dimers that fluoresce at $530 \mathrm{~nm}$ when the membrane potential is lost. The stained solution was also analyzed with flow cytometry. To detect the intracellular ROS level, the bacteria was stained with DCFH-DA $(20 \mu \mathrm{M})$, and analyzed using flow cytometry.

For TEM observation, bacterial samples in the control group and HMMP group were fixed with $2.5 \%$ glutaraldehyde at $4^{\circ} \mathrm{C}$ for $2 \mathrm{~h}$, then washed with PBS three times. Then, samples were serially dehydrated with ethanol at increased concentrations, permeated in the embedding medium and embedded for $48 \mathrm{~h}$ at $60^{\circ} \mathrm{C}$. Ultrathin sections of the embedded samples were prepared on the grids and stained with uranyl acetate. Finally, the grids were observed with TEM.

Phenotyping in vitro cultures of macrophages and BMDCs. Macrophages were seeded in 24-well plate at a cell density of 10,000 cells per well and cultured overnight. Then, the cells were further co-cultured with PBS, P, MP or HMMP $\left(20 \mu \mathrm{g} \mathrm{mL}^{-1}\right)$ for 8 hours, followed by US irradiation $\left(40 \mathrm{kHz}, 1.5 \mathrm{~W} \mathrm{~cm}^{-2}, 1\right.$ min per cycle, 5 cycles), then they were washed and harvested for further analysis. For macrophage polarization analysis, pellets were resuspended in $200 \mu \mathrm{L}$ of cold PBS with $1 \mu \mathrm{L}$ CD16/32 antibodies for Fc blocking, and were incubated at $4^{\circ} \mathrm{C}$ for $10 \mathrm{~min}$. Afterwards, the cells were incubated with PE/Cy7-labeled antiCD80 (16-10A1) for surface staining at $4^{\circ} \mathrm{C}$ in a dark enclosed space. After $30 \mathrm{~min}$. cells were washed and resuspended with PBS three times. To stain the intracellular marker, cells were fixed and permeabilized with Cyto-Fast ${ }^{\text {TM }}$ Fix/Perm Buffer Set following of the manufacturer's instructions, then stained with PE-labeled anti-CD206 (C068C2f) or another $30 \mathrm{~min}$, followed by flow cytometric analysis.

To assess the in vitro phagocytic clearance of bacteria by macrophages, the harvested macrophages were cultured with bacteria labelled with CFDA-SE in a ratio of $10: 1$ at $37^{\circ} \mathrm{C}$ in $5 \% \mathrm{CO}_{2}$ atmosphere. After coculture for $2 \mathrm{~h}$, the macrophages were spun down, and washed with PBS three times to remove bacteria in the supernatant. The phagocytosis of bacteria was then evaluated by flow cytometric analysis. For transcriptional analysis, total macrophage RNA was extracted with an Tizol (Invitrogen) followed by sequencing with DNBseq Platform (BGI-Wuhan, China). BMDCs were exposed to different treatments using the same protocol as macrophages except for the following tests. The BMDCs were incubated with CD16/32 for 10 min and further incubated with antibodies against CD11c (N418), CD80 (16-10A1) and CD86 (GL-1) under the guidance of the manufacturer's protocols. Lastly, BMDCs were washed with PBS three times and analyzed with flow cytometry. The gene expression levels of inflammatory related genes were analyzed with EZBioscience Qpcr kit (USA). RNA extraction and reverse transcription were performed according to the manufacturer's recommendations. The final quantitative PCR were performed on Roche LightCycler 480.

" In situ " osteomyelitis model establishment. To evaluate the direct therapeutic effect of our nanoplatform, we constructed "in situ" mouse osteomyelitis model using previous reported protocol ${ }^{6}$ with 
some modifications. The male C57 mice (6 weeks old) were allocated to four groups randomly, naming as control, P, MP and HMMP. The mice were deeply anesthetized by intraperitoneal injection of pentobarbital. After anesthesia induction, the left leg of the mice was manually depilated and disinfected. The knee joint was exposed after layer-by-layer incisions, then a bone channel was created in the tibial plateau with a medical electric drill. $50 \mu \mathrm{L}$ of $10^{6} \mathrm{CFU} \mathrm{mL}^{-1} \mathrm{~S}$. aureus were injected into the channel followed by the placement of a custom-made implant. The wound was then carefully sutured layer-bylayer. Then the mouse was housed carefully for 1 day for the development of osteomyelitis. Treatment was initiated $24 \mathrm{~h}$ after the induction of infection, the mice were treated with $100 \mu \mathrm{L}$ of either PBS, P, MP or HMMP ( $\left.2 \mathrm{mg} \mathrm{mL}^{-1}\right)$ via intravenous injection, and then irradiated with US $\left(40 \mathrm{kHz}, 1.5 \mathrm{~W} \mathrm{~cm}^{-2}, 1 \mathrm{~min}\right.$ per cycle, 5 cycles). To assess the therapeutic effect of different treatments, general observations, clinical imaging assessment, and bacterial viability test were performed at predetermined time points. Knee perimeter was recorded over time with a caliper and quantified using the formula of knee perimeter $=\pi \times$ $a \times b$, where $a$ and $b$ are the large and small diameter, respectively. Infection severity was monitored using 3.0 T MR scanner MRI (CG NOVILA 7.0T). 21 days after the surgery, the implant were extracted and periimplant tissues were dissected for bacterial CFU assay. The explanted tibias were fixed and decalcified in EDTA for 2 weeks, pathological sections were then prepared for Gimesa staining. Immunofluorescence staining was also performed for CD45, iNOS and CD206.

The both ends of the tibia were cut, and bone marrow suspensions were collected using an injector. The obtained bone marrow suspensions were digested in dissociation buffer $\left(100 \mu \mathrm{g} \mathrm{mL}^{-1}\right.$ deoxyribonuclease and $1 \mathrm{mg} \mathrm{mL}^{-1}$ collagenase IV) at $37^{\circ} \mathrm{C}$ for $30 \mathrm{~min}$. The cell suspensions were then mashed over $70 \mu \mathrm{m}$ strainers to obtain single cell suspension. Erythrocytes were depleted with ACK lysis buffer (Gibco) and washed with ice-cold PBS three times. For RNA-seq analysis, the total RNA was extracted with Trizol and followed by sequencing with DNBseq Platform. For the analysis of immune cells composition, the cell suspensions were incubated with CD16/32 for $10 \mathrm{~min}$ and then stained with antibodies against CD45 (30-F11), CD3 (145-2C11), CD4 (GK1.5), CD8 (53-6.7), F4/80 (BM8), CD80 (16-10A1), CD86 (GL-1), I-A/I-E (M5/114.15.2), CD11c (N418), CD19 (6D5), CD138 (281-2), NK1.1 (PK136), CD11b (M1/70), and Gr1 (RB6-8C5) on the ice for $30 \mathrm{~min}$. The CD206 staining protocol is the same as that used in vitro. After surface staining, the cell suspensions were fixed and permeabilized with True-Nuclear $^{\text {TM }}$ Transcription Factor Buffer Set, then stained with antibodies against Foxp3 for 30 min on the ice in a dark enclosed place. After staining, the cell suspensions were centrifuged and washed with ice-cold PBS thrice, then resuspended in $500 \mu \mathrm{L}$ ice-cold PBS for flow analysis. The cytokines (IL-10, TNFa, IL-12 and IFN- $\gamma$ ) and antibodies (IgM and IgG) in the bone marrow were tested with ELISA kits according to the manufacturer's recommendations.

In vivo monitoring of systemic immune responses. Contralateral mouse osteomyelitis model was established to evaluate the systematic antibacterial immune responses as follows. First, we established an "in situ" osteomyelitis model in the left knee and treated with different methods as above mentioned. 14 days later, osteomyelitis model was established on the right knee using the same method as above. Again, the evaluation approaches for infection severity such as general observation, MRI imaging, 
bacterial viability test and Gimesa staining described above was used. Procedures for isolating and staining spleen-associated immune cells were similar to the protocols used for bone marrow. Briefly, the spleens were dissected and cut into small pieces, enzymatically degraded, and mechanically disrupted 70 $\mu \mathrm{m}$ cell strainers. After red blood cell removal with ACK lysis buffer, the cell suspensions were incubated with CD16/32 for 10 min and then stained with antibodies against CD45 (30-F11), CD3 (145-2C11), CD4 (GK1.5), CD8 (53-6.7), CD19 (6D5), CD138 (281-2), NK1.1 (PK136), CD11b (M1/70), and Gr1 (RB6-8C5) on the ice according to the manufacturer's instructions. The intracellular staining of Foxp3 (MF-14) was the same as the procedures for bone marrow. Serum cytokines (IL-10 and TNFa) and antibody levels (IgM and $\lg \mathrm{G}$ ) were determined with Elisa kits following the manufacturer's instruction.

Prophylactic vaccine study. To determine whether HMMP vaccine could elicit long-term immune memory responses or not, in 21 days after the establishment of in situ osteomyelitis model, we undertook thorough debridement, implant removal and local vancomycin (1 mg, Sangon Biotech) administration. After the surgery, $15 \mathrm{mg} \mathrm{kg}^{-1}$ of vancomycin was administrated intravenously per day. The procedures fully mimic the clinical management of osteomyelitis. 7 days later, after the infection was controlled, the mice received a re-challenge surgery in a manner identical to that in the "in situ" osteomyelitis model. The data of general photos, knee perimeter, MRI imaging were collected at preset time after the surgery. In 21 days after the surgery, the mice were euthanized. Bacterial viability test in vivo and Gimesa staining of the infected bone were conducted with above mentioned methods. The bone and spleen were harvested, and processed into single-cell suspension as above. Then bone marrow single-cell suspensions were stained with antibodies against B220 (RA3-6B2), IgG (Poly4053) and IgD (11-26c.2a), while the spleen single-cell suspensions were stained with antibodies against CD3 (145-2C11), CD4 (GK1.5), CD8 (53-6.7), CD44 (IM7), and CD62L (MEL-14), according to the manufacturer's instructions.

\section{Declarations}

\section{Acknowledgements}

We greatly acknowledge the National Natural Science Foundation of China (Grant No. 52002391, 81772309, 81974324 and 21835007), Key Research Program of Frontier Sciences, Chinese Academy of Sciences (Grant No. ZDBS-LY-SLH029), China Postdoctoral Science Foundation (Grant No. 2019M660098), and Science Foundation for Youth Scholar of State Key Laboratory of High Performance Ceramics and Superfine Microstructures (grant no. SKL201906).

\section{References}

1. Lew, D.P. \& Waldvogel, F.A. Osteomyelitis. Lancet 364, 369-379 (2004).

2. Kapadia, B.H. et al. Periprosthetic joint infection. Lancet 387, 386-394 (2016).

3. Kavanagh, N. et al. Staphylococcal Osteomyelitis: Disease Progression, Treatment Challenges, and Future Directions. Clin. Microbiol. Rev. 31 (2018). 
4. Li, J. et al. Zinc-doped Prussian blue enhances photothermal clearance of Staphylococcus aureus and promotes tissue repair in infected wounds. Nat. Commun. 10, 4490 (2019).

5. Galstyan, A., Schiller, R. \& Dobrindt, U. Boronic Acid Functionalized Photosensitizers: A Strategy To Target the Surface of Bacteria and Implement Active Agents in Polymer Coatings. Angew. Chem., Int. Ed. Engl. 56, 10362-10366 (2017).

6. Qiao, Y. et al. Treatment of MRSA-infected osteomyelitis using bacterial capturing, magnetically targeted composites with microwave-assisted bacterial killing. Nat. Commun. 11, 4446 (2020).

7. Singh, K.S. et al. IspH inhibitors kill Gram-negative bacteria and mobilize immune clearance. Nature (2020).

8. Jain, N. et al. Mesenchymal stem cells offer a drug-tolerant and immune-privileged niche to Mycobacterium tuberculosis. Nat. Commun. 11, 3062 (2020).

9. Finlay, B.B. \& McFadden, G. Anti-immunology: evasion of the host immune system by bacterial and viral pathogens. Cell 124, 767-782 (2006).

10. Rosenberger, C.M. \& Finlay, B.B. Phagocyte sabotage: disruption of macrophage signalling by bacterial pathogens. Nat. Rev. Mol. Cell. Biol. 4, 385-396 (2003).

11. Scharping, N.E. et al. Mitochondrial stress induced by continuous stimulation under hypoxia rapidly drives T cell exhaustion. Nat. Immunol. (2021).

12. Hotchkiss, R.S., Monneret, G. \& Payen, D. Sepsis-induced immunosuppression: from cellular dysfunctions to immunotherapy. Nat. Rev. Immunol. 13, 862-874 (2013).

13. $\mathrm{Xu}, \mathrm{J}$. et al. A general strategy towards personalized nanovaccines based on fluoropolymers for postsurgical cancer immunotherapy. Nat. Nanotechnol. 15, 1043-1052 (2020).

14. Cifuentes-Rius, A., Desai, A., Yuen, D., Johnston, A.P.R. \& Voelcker, N.H. Inducing immune tolerance with dendritic cell-targeting nanomedicines. Nat. Nanotechnol. 16, 37-46 (2021).

15. Conniot, J. et al. Immunization with mannosylated nanovaccines and inhibition of the immunesuppressing microenvironment sensitizes melanoma to immune checkpoint modulators. Nat. Nanotechnol. 14, 891-901 (2019).

16. Li, L. et al. Burst release of encapsulated annexin A5 in tumours boosts cytotoxic T-cell responses by blocking the phagocytosis of apoptotic cells. Nat. Biomed. Eng. 4, 1102-1116 (2020).

17. Singh, A. Eliciting B cell immunity against infectious diseases using nanovaccines. Nat. Nanotechnol. 16, 16-24 (2021).

18. Rousset, $F$. et al. The impact of genetic diversity on gene essentiality within the Escherichia coli species. Nat. Microbiol. (2021).

19. Bakaletz, L.O. Developing animal models for polymicrobial diseases. Nat. Rev. Microbiol. 2, 552-568 (2004).

20. Zhang, Q. et al. Neutrophil membrane-coated nanoparticles inhibit synovial inflammation and alleviate joint damage in inflammatory arthritis. Nat. Nanotechnol. 13, 1182-1190 (2018). 
21. Zhou, J., Kroll, A.V., Holay, M., Fang, R.H. \& Zhang, L. Biomimetic Nanotechnology toward Personalized Vaccines. Adv. Mater. 32, e1901255 (2020).

22. Wang, C. et al. Pretreated Macrophage-Membrane-Coated Gold Nanocages for Precise Drug Delivery for Treatment of Bacterial Infections. Adv. Mater. 30 (2018).

23. Fusciello, M. et al. Artificially cloaked viral nanovaccine for cancer immunotherapy. Nat. Commun. 10, 5747 (2019).

24. Rao, L. et al. Hybrid cellular membrane nanovesicles amplify macrophage immune responses against cancer recurrence and metastasis. Nat. Commun. 11, 4909 (2020).

25. Yang, G. et al. Hollow $\mathrm{MnO}_{2}$ as a tumor-microenvironment-responsive biodegradable nano-platform for combination therapy favoring antitumor immune responses. Nat. Commun. 8, 902 (2017).

26. Son, S. et al. Multifunctional sonosensitizers in sonodynamic cancer therapy. Chem. Soc. Rev. 49, 3244-3261 (2020).

27. Lv, M. et al. Manganese is critical for antitumor immune responses via cGAS-STING and improves the efficacy of clinical immunotherapy. Cell. Res. 30, 966-979 (2020).

28. Fluckiger, A. et al. Cross-reactivity between tumor MHC class I-restricted antigens and an enterococcal bacteriophage. Science 369, 936-942 (2020).

29. Hu, C.M. et al. Nanoparticle biointerfacing by platelet membrane cloaking. Nature 526, 118-121 (2015).

30. Sun, H. et al. Cancer-Cell-Biomimetic Nanoparticles for Targeted Therapy of Homotypic Tumors. Adv. Mater. 28, 9581-9588 (2016).

31. Akkaya, M., Kwak, K. \& Pierce, S.K. B cell memory: building two walls of protection against pathogens. Nat. Rev. Immunol. 20, 229-238 (2020).

32. Liang, S., Deng, X., Ma, P., Cheng, Z. \& Lin, J. Recent Advances in Nanomaterial-Assisted Combinational Sonodynamic Cancer Therapy. Adv. Mater. 32, e2003214 (2020).

33. Roewe, J. et al. Bacterial polyphosphates interfere with the innate host defense to infection. Nat. Commun. 11, 4035 (2020).

34. Shields, C.W.t. et al. Cellular backpacks for macrophage immunotherapy. Sci. Adv. 6, eaaz6579 (2020).

35. Williford, J.M. et al. Recruitment of CD103(+) dendritic cells via tumor-targeted chemokine delivery enhances efficacy of checkpoint inhibitor immunotherapy. Sci. Adv. 5, eaay1357 (2019).

36. Smith, L.K. et al. beta2-microglobulin is a systemic pro-aging factor that impairs cognitive function and neurogenesis. Nat. Med. 21, 932-937 (2015).

37. Carreno, B.M. et al. Cancer immunotherapy. A dendritic cell vaccine increases the breadth and diversity of melanoma neoantigen-specific T cells. Science 348, 803-808 (2015).

38. Moyer, T.J. et al. Engineered immunogen binding to alum adjuvant enhances humoral immunity. Nat. Med. 26, 430-440 (2020). 
39. Bohannon, C. et al. Long-lived antigen-induced IgM plasma cells demonstrate somatic mutations and contribute to long-term protection. Nat. Commun. 7, 11826 (2016).

40. Baumann, T. et al. Regulatory myeloid cells paralyze T cells through cell-cell transfer of the metabolite methylglyoxal. Nat. Immunol. 21, 555-566 (2020).

41. Hoetzenecker, W. et al. ROS-induced ATF3 causes susceptibility to secondary infections during sepsis-associated immunosuppression. Nat. Med. 18, 128-134 (2011).

42. van der Poll, T., van de Veerdonk, F.L., Scicluna, B.P. \& Netea, M.G. The immunopathology of sepsis and potential therapeutic targets. Nat. Rev. Immunol. 17, 407-420 (2017).

43. Voll, R.E. et al. Immunosuppressive effects of apoptotic cells. Nature 390, 350-351 (1997).

44. Mesin, L. et al. Restricted Clonality and Limited Germinal Center Reentry Characterize Memory B Cell Reactivation by Boosting. Cell 180, 92-106 e111 (2020).

45. Bencherif, S.A. et al. Injectable cryogel-based whole-cell cancer vaccines. Nat. Commun. 6, 7556 (2015).

46. Ci, T. et al. Cryo-shocked cancer cells for targeted drug delivery and vaccination. Sci. Adv. 6 (2020).

47. Wang, H. et al. Biomaterial-based scaffold for in situ chemo-immunotherapy to treat poorly immunogenic tumors. Nat. Commun. 11, 5696 (2020).

48. Lizotte, P.H. et al. In situ vaccination with cowpea mosaic virus nanoparticles suppresses metastatic cancer. Nat. Nanotechnol. 11, 295-303 (2016).

49. Chen, Q. et al. Photothermal therapy with immune-adjuvant nanoparticles together with checkpoint blockade for effective cancer immunotherapy. Nat. Commun. 7, 13193 (2016).

\section{Figures}


a
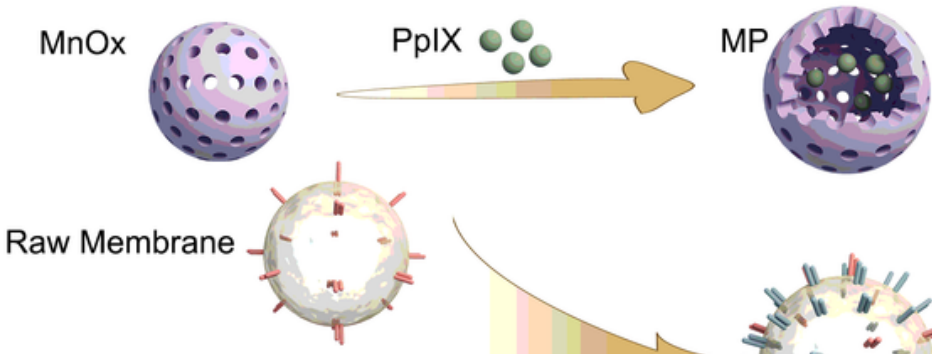

4T1 Membrane
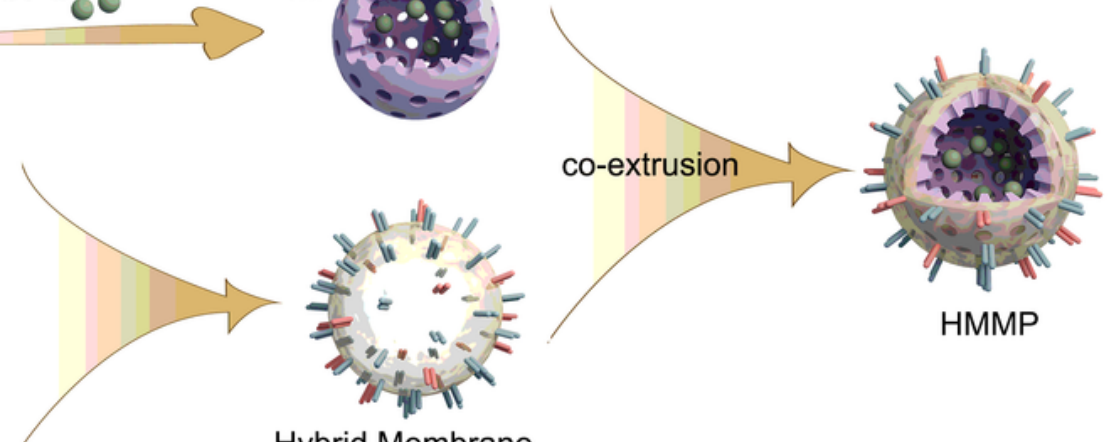

Hybrid Membrane
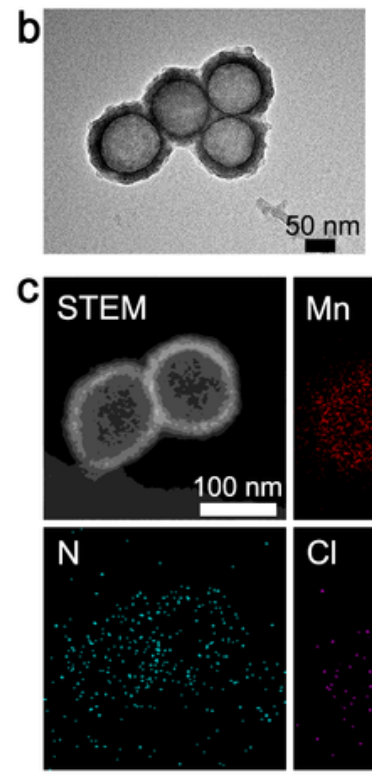

e

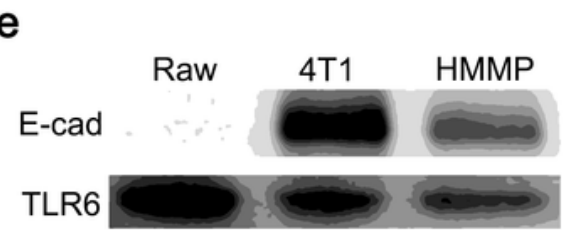

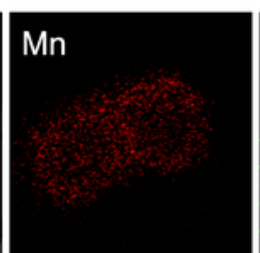

$\mathrm{Cl}$

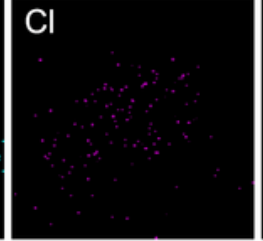

$\mathrm{P}$

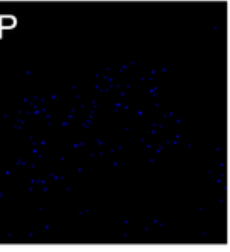

g
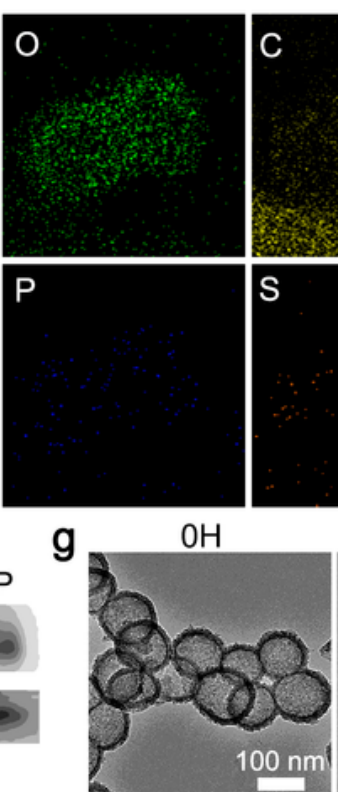

s
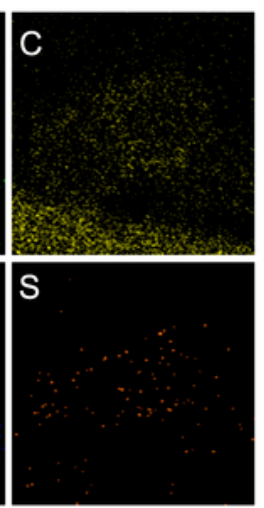

$3 \mathrm{H}$
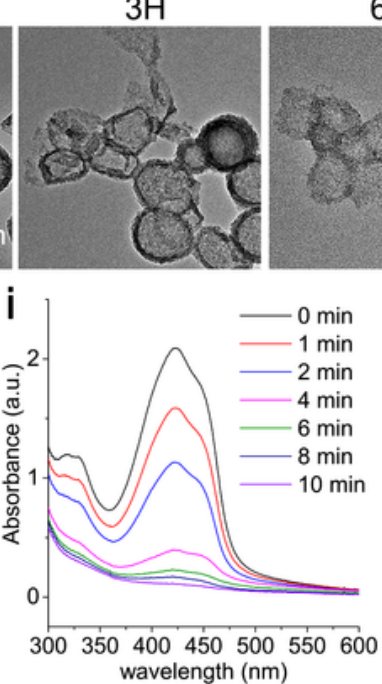

100

75

60

45

35 d Marker MnOx Raw 4T1 HMMP $\mathrm{kDa}$

180

140

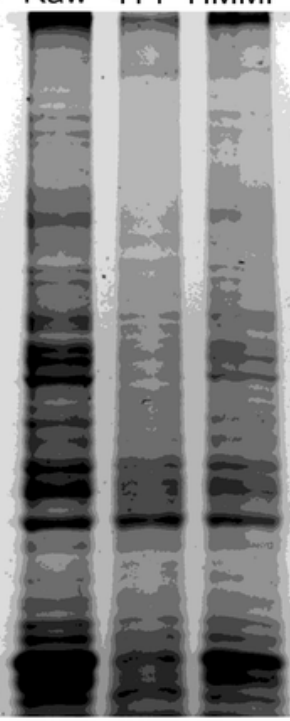

$6 \mathrm{H}$

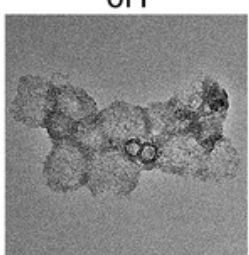

$12 \mathrm{H}$

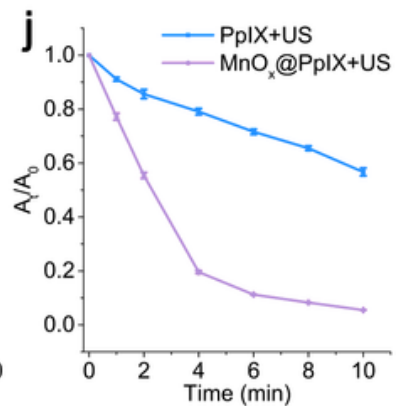

\section{Figure 1}

Schematics of HMMP construction and characterization. a, Schematic illustration for the construction of HMMP. b, Representative TEM images of HMMP at varied magnifications. Samples were stained with uranyl acetate. c, Representative HAADF-STEM image and element-mapping images of HMMP. d, Overall protein bands of MnOx, RAW264.7 macrophage membrane-derived vesicles (MV), 4T1 tumor membranederived vesicles and HMMP resolved by SDS-page and Coomassie Brilliant Blue staining. e, Characteristic 
protein bands of RAW264.7 macrophage membrane-derived vesicles, 4T1 tumor membrane-derived vesicles and HMMP resolved by western blotting. $f$, Diameter and zeta potential measurements. $g$, Representative TEM images of HMMP after incubation in $4 \mathrm{mM}$ of GSH. h, Representative TEM images of MnOx after incubation in $4 \mathrm{mM}$ of GSH. i, Time-dependent oxidation of DPBF indicating ROS generation by MnOx@PpIX under US irradiation. j, Comparison of DPBF oxidation by PpIX and MnOx@PpIX under US irradiation for 10 minutes.
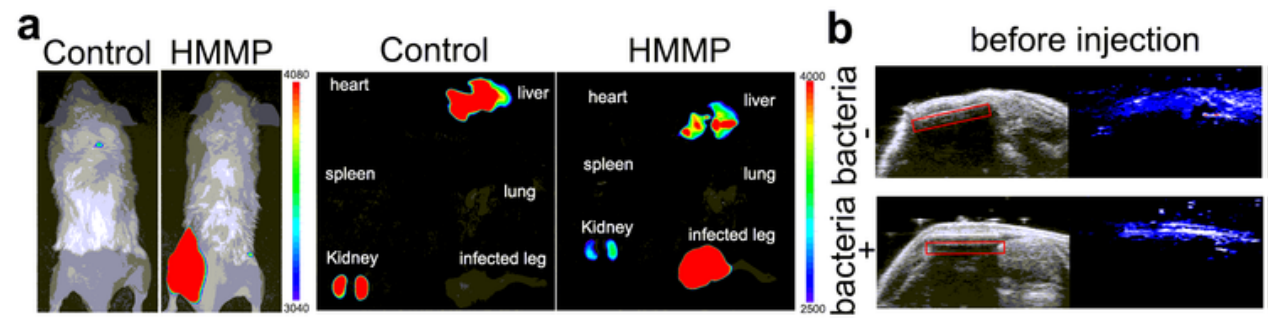
60min post-injection
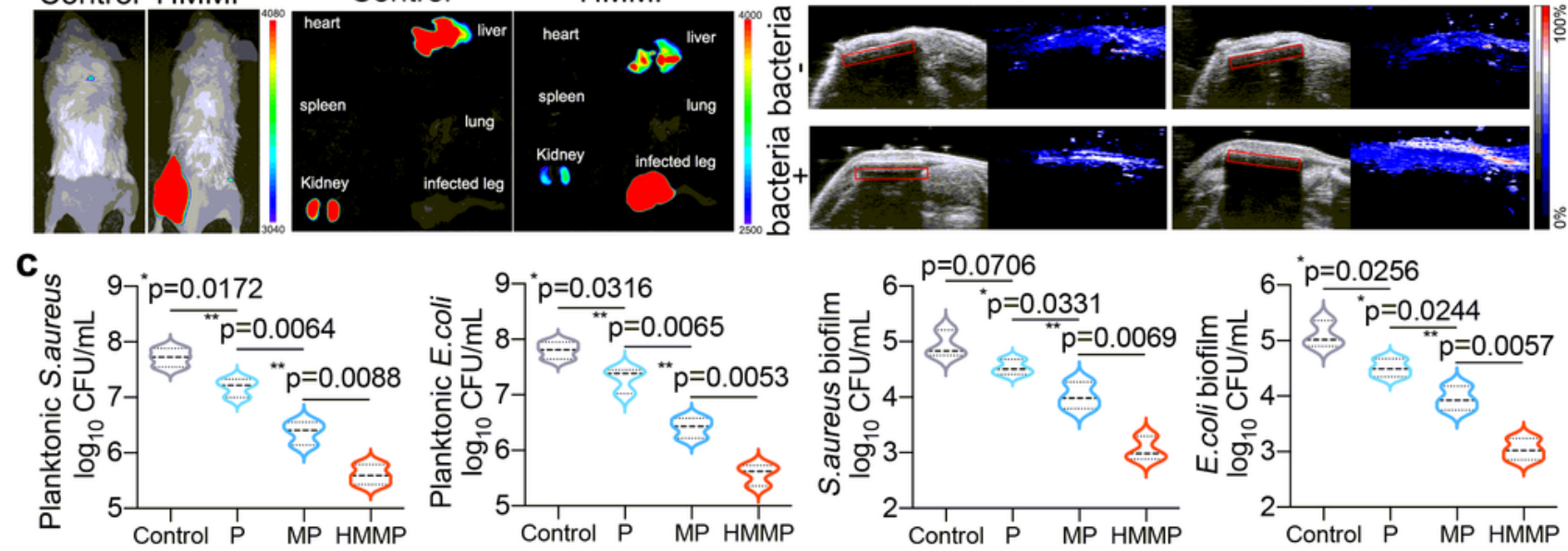

d
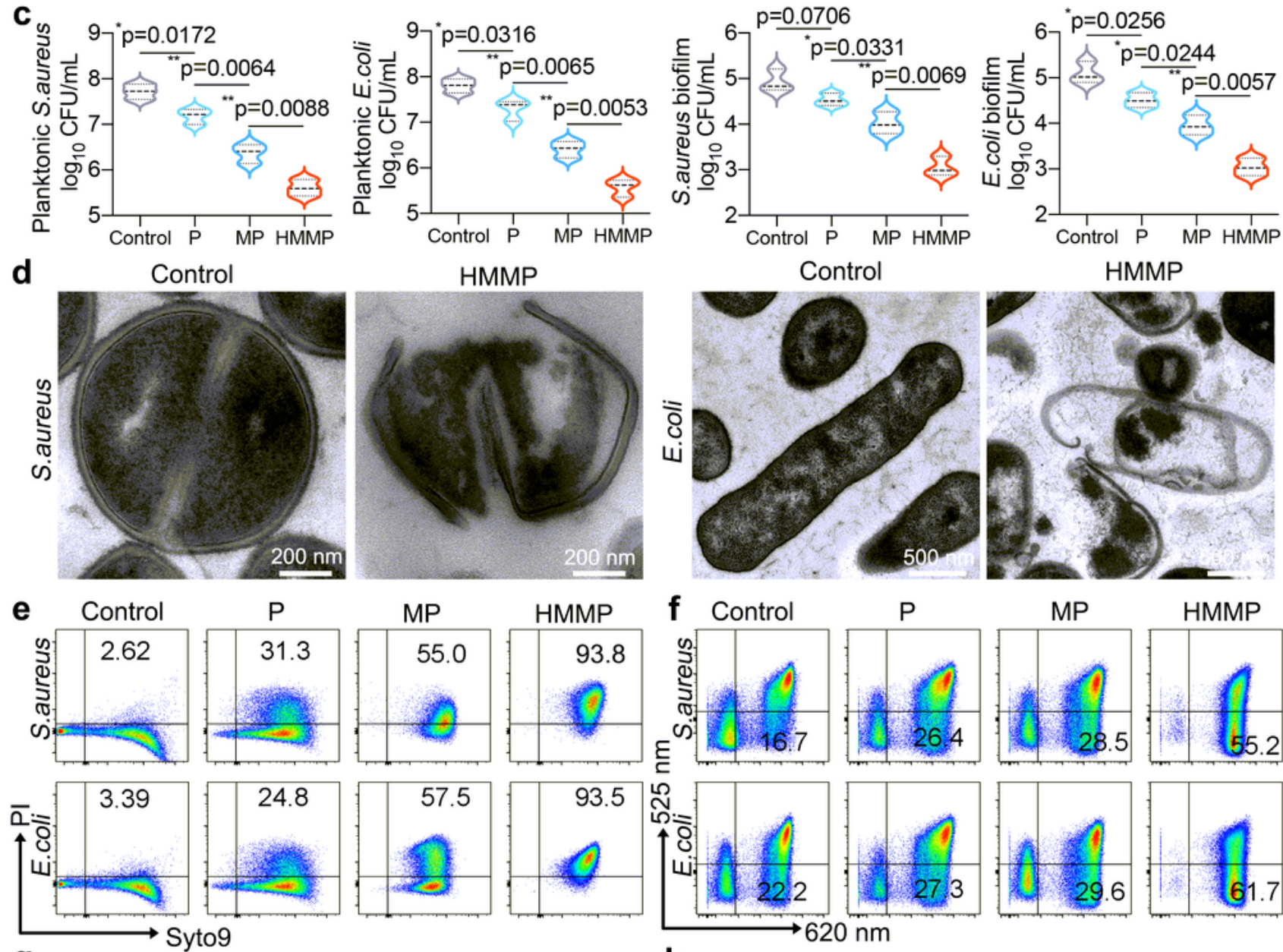

g
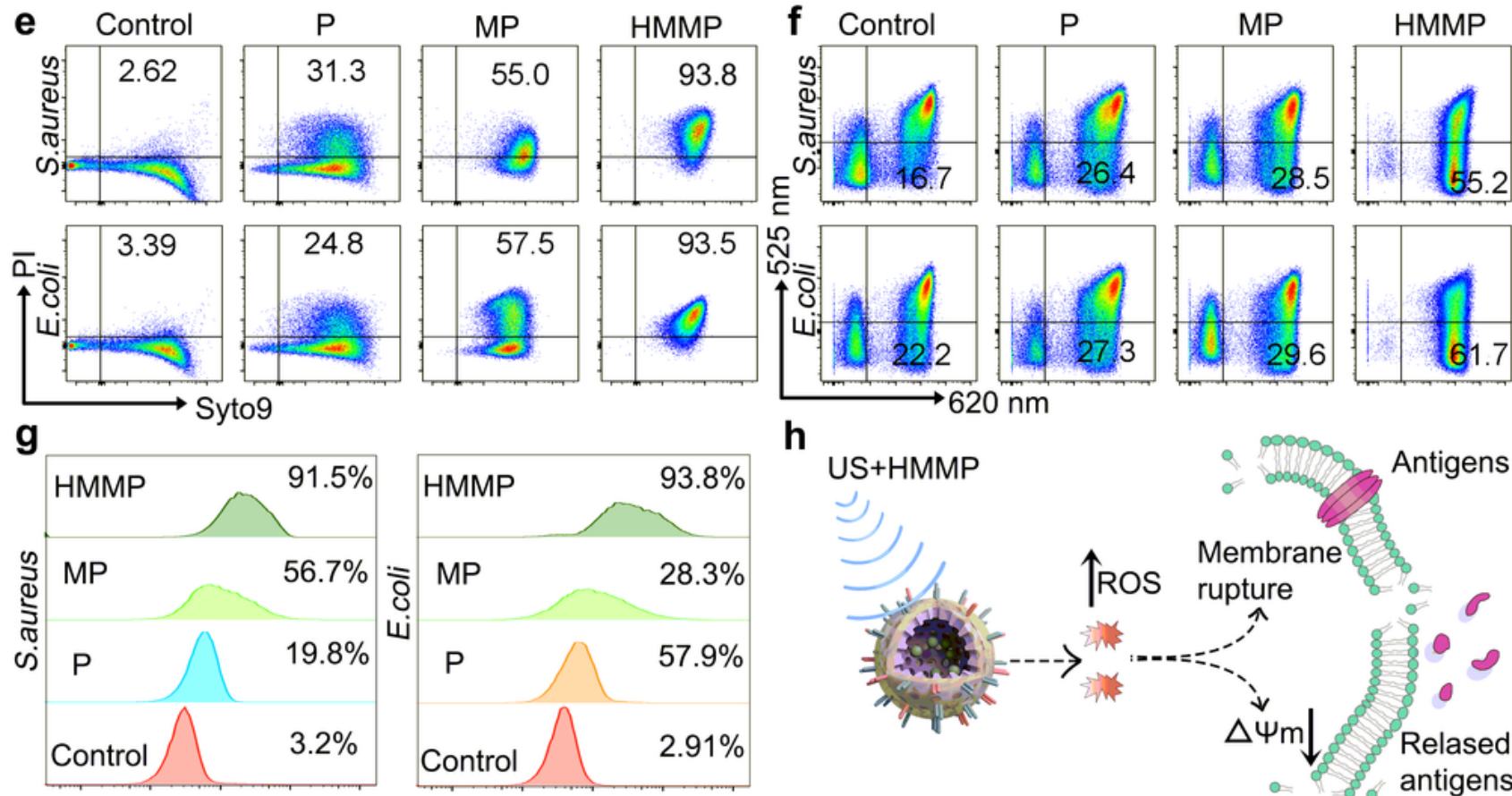

h
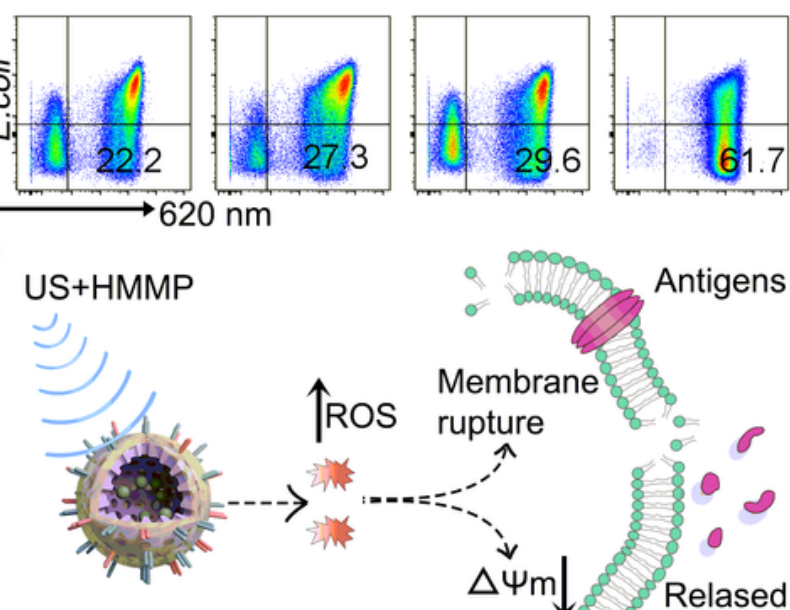

Figure 2 
HMMP triggers burst bacterial antigen release. a, In vivo and Ex vivo fluorescence images of osteomyelitis-bearing mice taken in $24 \mathrm{~h}$ after the injection of Cy5.5 labeled HMMP intravenously. b, Oxygen levels in the subcutaneous implant model detected by PA imaging after intravenous HMMP injection (the red rectangular indicates implant). c, Planktonic and biofilm bacterial CFU count after different treatments. d, Representative bacterial TEM images in control and HMMP treated groups. e, Representative flow cytometry plots of live/dead staining. f, Representative flow cytometry plots of DiOC2 staining. g, Representative flow cytometry data of DCFH-DA staining to show intracellular ROS levels. h, Schematic illustration of a possible mechanism of HMMP triggered bacterial antigen release. $\triangle \Psi \mathrm{m}$, membrane potential. 

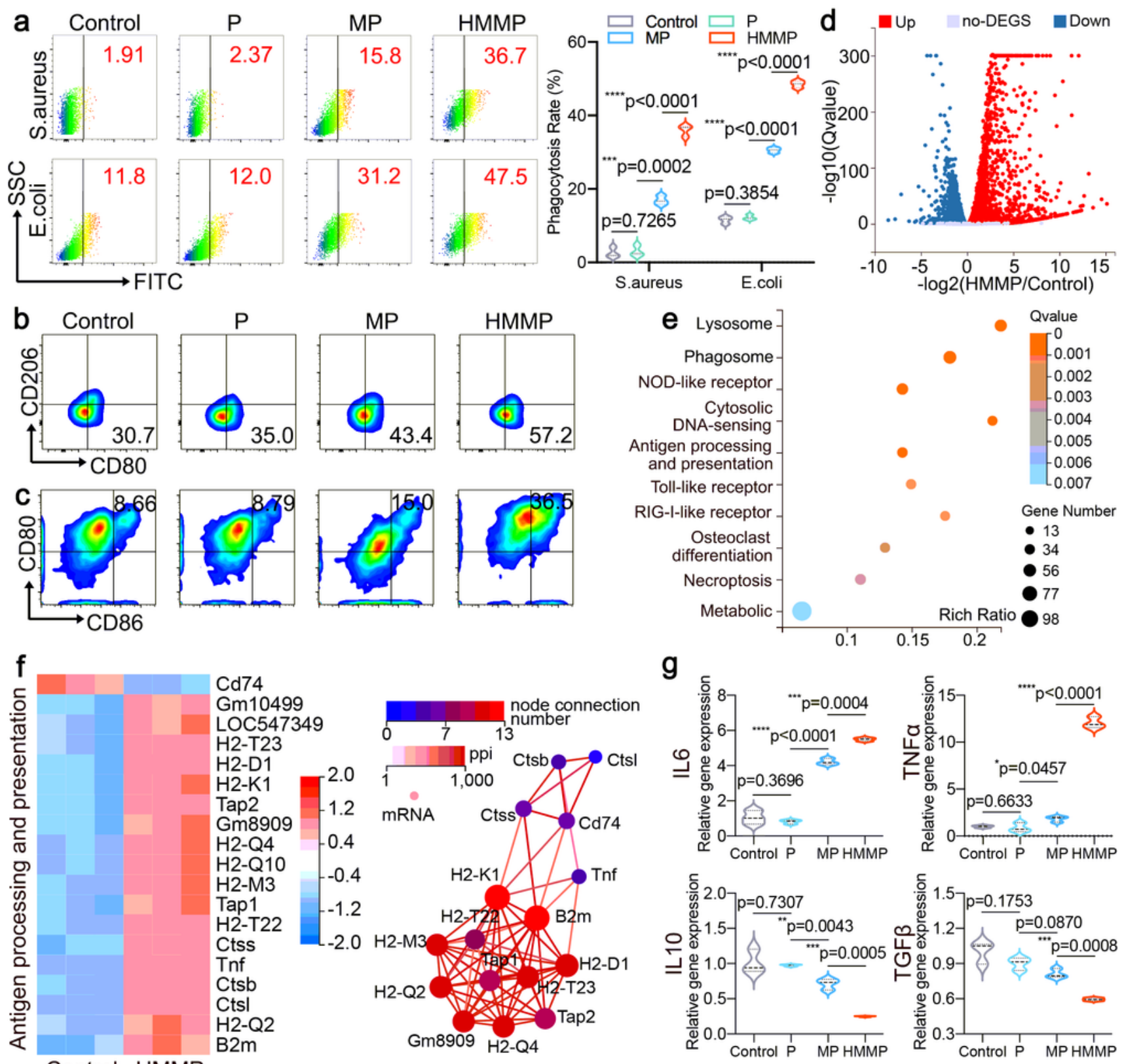

Control HMMP

h immature DC mature DC antigen processing and presentation

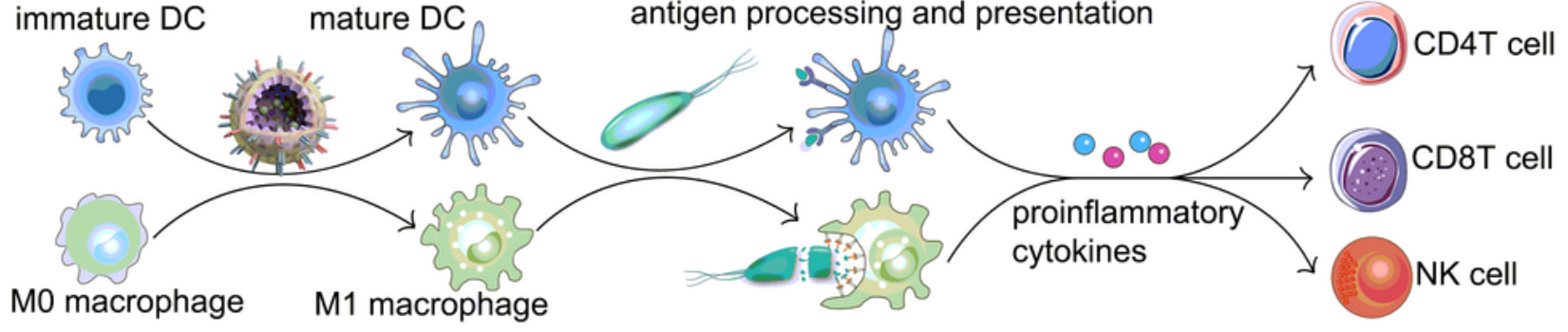

\section{Figure 3}

HMMP promotes effective bacterial phagocytosis and antigen processing/presentation by APCs. a, Representative flow cytometry plots and quantification of bacterial phagocytosis rate by macrophages. b,c, Representative flow cytometry plots to show macrophage polarization (b) and BMDCs maturation (c) induced by different treatments in vitro. $d$-f, Transcriptomic analysis of macrophages co-cultured with HMMP or PBS (Control). d, Vocano plots of DEGs in the HMMP and control group. e, KEGG enrichment 
analysis of the DEGs in the HMMP and control groups $f$, Gene signature analysis of DEGs in antigen processing and presentation pathway in the macrophages after different treatments. $\mathrm{n}=3$ biologically independent samples per group. g, Qpcr gene analysis of BMDCs after different treatments in vitro. $h$, Schematic illustration of HMMP induced APCs priming.
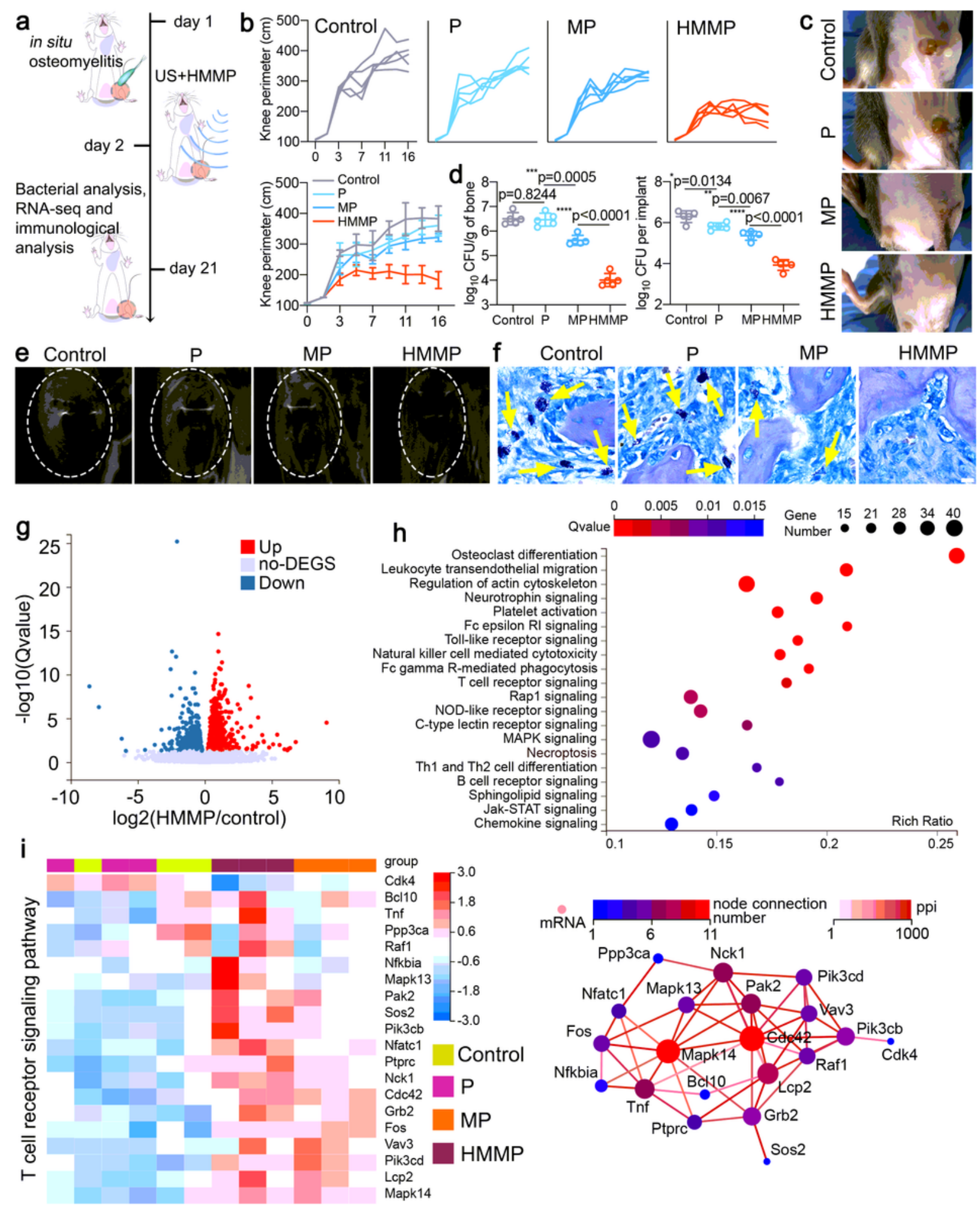

Figure 4 
HMMP in situ nanovaccination inhibits bacterial growth and changes the transcriptomic profiles in the established osteomyelitis. a, Schematic illustration of the osteomyelitis model establishment. b, Individual and average knee perimeter curves of the infected knee. c, Digital photos of the infected knee in 21 days after the primary surgery. $d$, The MRSA CFU counts in the infected bone marrow and extracted implant. e, MRI imaging of the infected knee. The white circle indicates the infected knee. $f$, Gimesa staining images of infected bone marrow. The yellow arrows indicate S. aureus infection in deep blue. Scale bars, $10 \mu \mathrm{m}$. g-i, Transcriptomic analysis of the infected bone marrow tissues from mice after different treatments. g, Vocano plots of DEGs in the HMMP and control group. h, KEGG enrichment analysis of the DEGs in the HMMP and control group. i, Gene signature analysis of DEGs in T cell receptor signaling pathway in the bone marrow from mice after different treatments. $n=3$ biologically independent mice per group. 


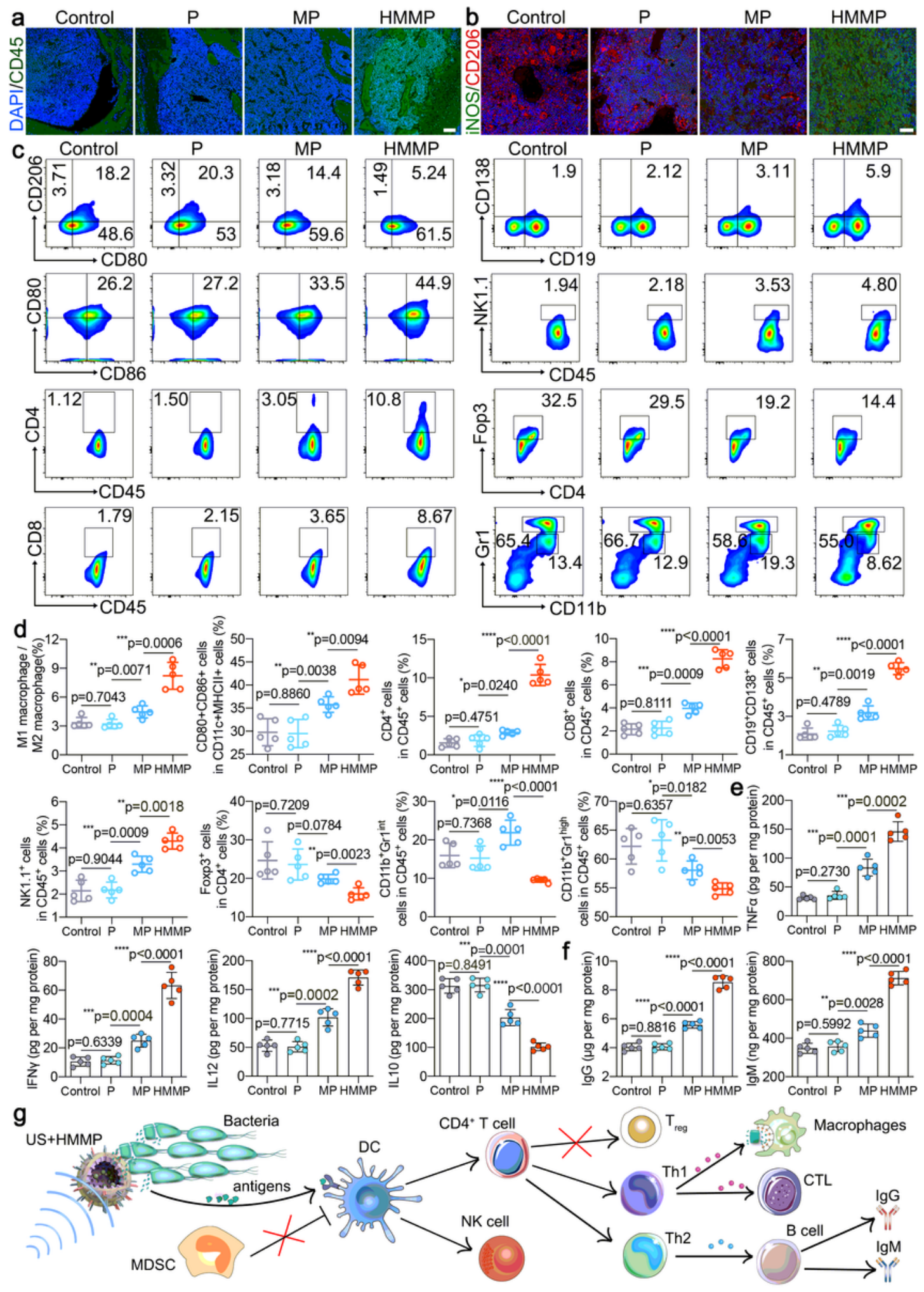

Figure 5

HMMP in situ vaccination recruits and activates abundant immune cells. a, Representative immunofluorescence images of CD45+ cells in the bone marrow. Scale bars, $75 \mu \mathrm{m}$. b, Representative immunofluorescence images of iNOS+ and CD206+ cells in the bone marrow. Scale bars, $50 \mu \mathrm{m} . \mathrm{c}, \mathrm{d}$, Representative flow cytometry plots (c) and quantification (d) of M1-polarized macrophages (CD80+CD206-) after gating on CD11b+F4/80+ cells, mature DCs (CD80+CD86+) after gating on 
CD11 c+l-A/I-E+ cells, CD4+ T cells after gating on CD45+ cells, CD8+ T cells after gating on CD45+ cells, plasma cells (CD138+) after gating on CD45+CD19+ cells, NK cells (NK1.1+) after gating on CD45+ cells, Treg cells (FoxP3+) after gating on CD3+CD4+ cells, granulocytic MDSCs (Gr1highCD11b+) and monocytic MDSCs (Gr1 intCD11b+) after gating on CD45+ in the bone marrows on day 21 after treatments. $n=5$ per group. In flow cytometry plots, percentage of cells in the adjacent bound area is indicated. e, TNFa, IFNY, IL12 and IL10 concentrations in the bone marrow. f, IgG and IgM concentrations in the bone marrow. $\mathrm{g}$, Schematic illustration of the in situ vaccination process.
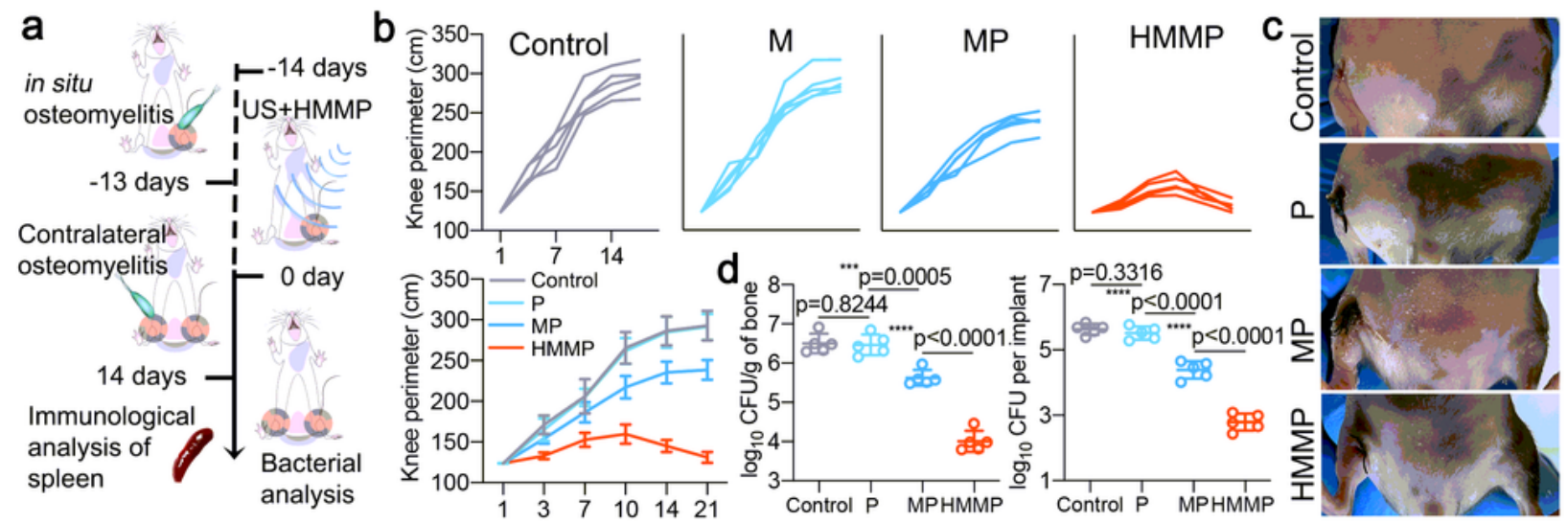

e Control
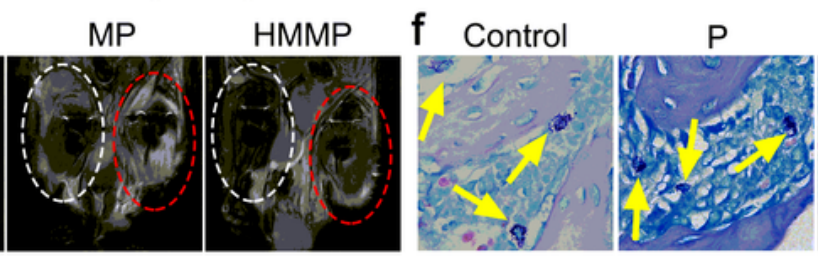

$M P$

HMMP
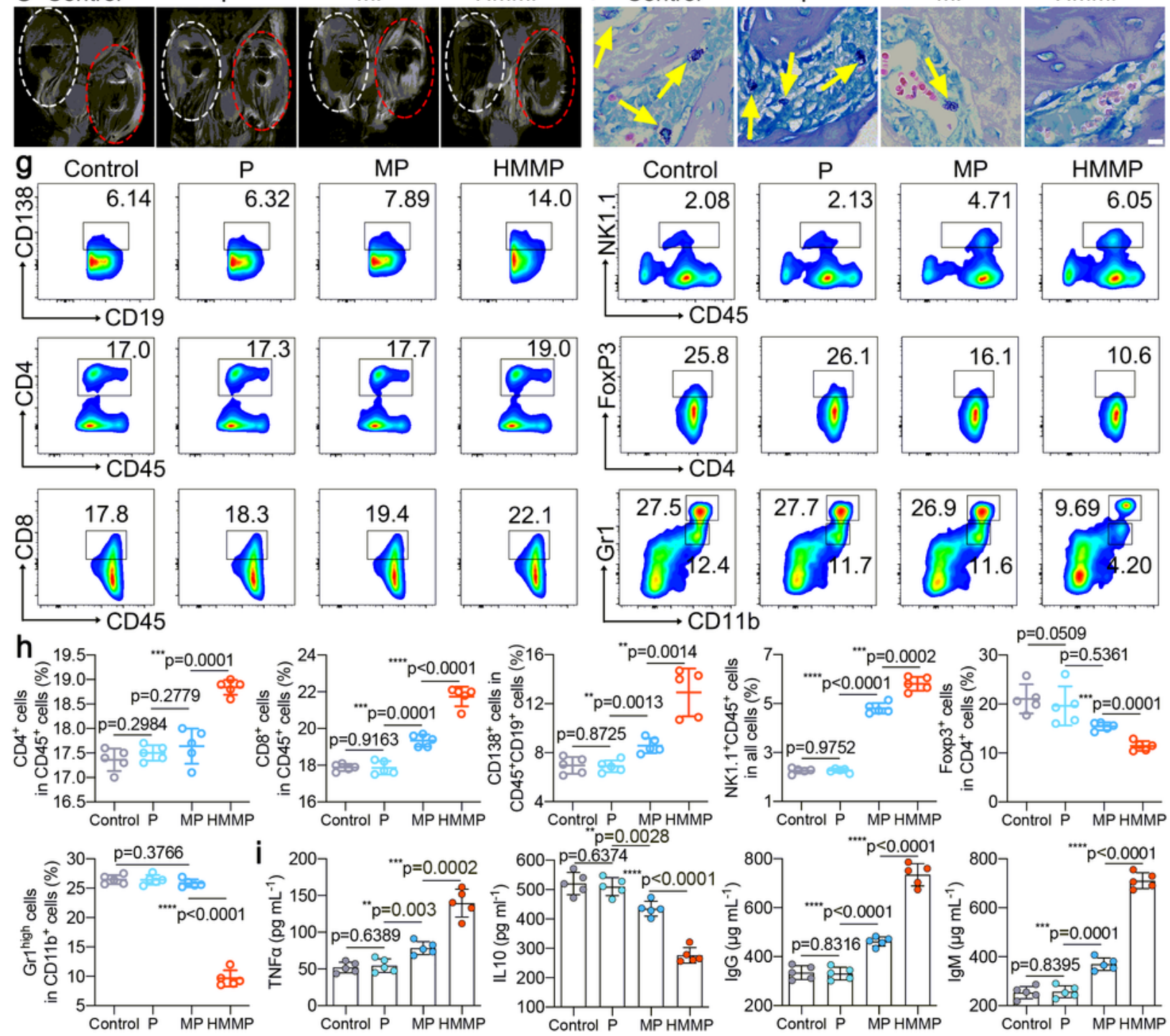


\section{Figure 6}

HMMP in situ vaccination inflames systemic antibacterial immune responses against contralateral osteomyelitis. a, Scheme and timeline of the experimental design to evaluate the systemic immune responses triggered by HMMP in situ vaccination. $b$, Individual and average knee perimeter curves of the contralateral infected knee. c, Digital photos of the infected knee in 14 days after the construction of the contralateral osteomyelitis model by secondary surgery. $d$, The MRSA CFU counts in the infected bone marrow and extracted implant in 14 days after the second surgery. e, MRI imaging of both sides of the infected knees. The white and red circle indicate the first and secondary osteomyelitis, respectively. f, Gimesa staining images of infected bone marrow. The yellow arrows indicate S. aureus infections in deep blue. Scale bars, $10 \mu \mathrm{m}$. g,h, Representative flow cytometry plots (g) and quantification (h) of plasma cells (CD138+) after gating on CD45+CD19+ cells, CD4+ T cells after gating on CD45+ cells, CD8+ T cells after gating on CD45+ cells, NK cells (NK1.1+) after gating on CD45+ cells, Treg cells (FoxP3+) after gating on CD3+CD4+ cells, granulocytic MDSCs (Gr1highCD11b+) and monocytic MDSCs (Gr1 intCD11b+) after gating on CD 45+ in the spleens 14 days after the secondary osteomyelitis. $n=5$ per group. In flow cytometry plots, percentage of cells in the adjacent bound area is indicated. i, TNFa, IL10, $\lg \mathrm{G}$ and IgM concentrations in the serum. 

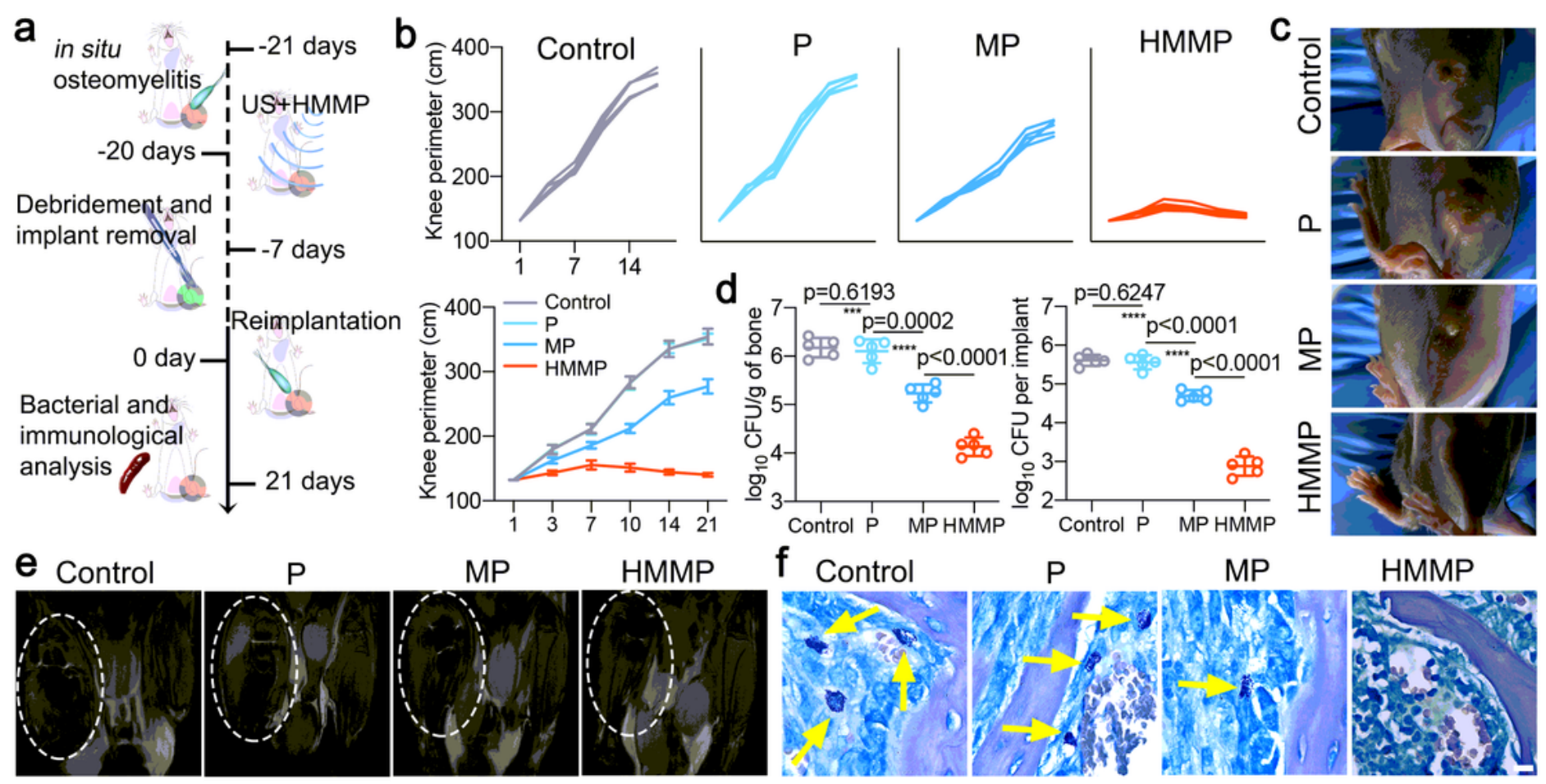

HMMP
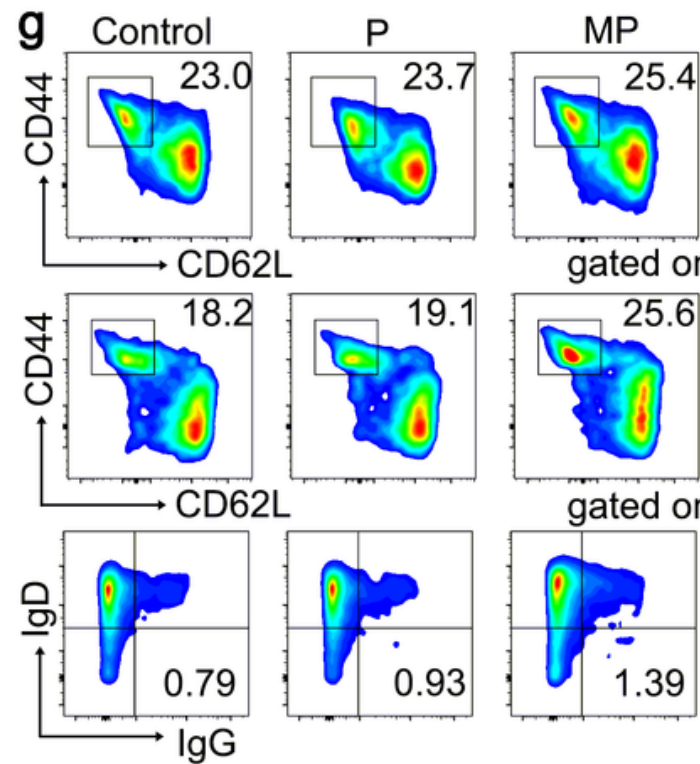

HMMP
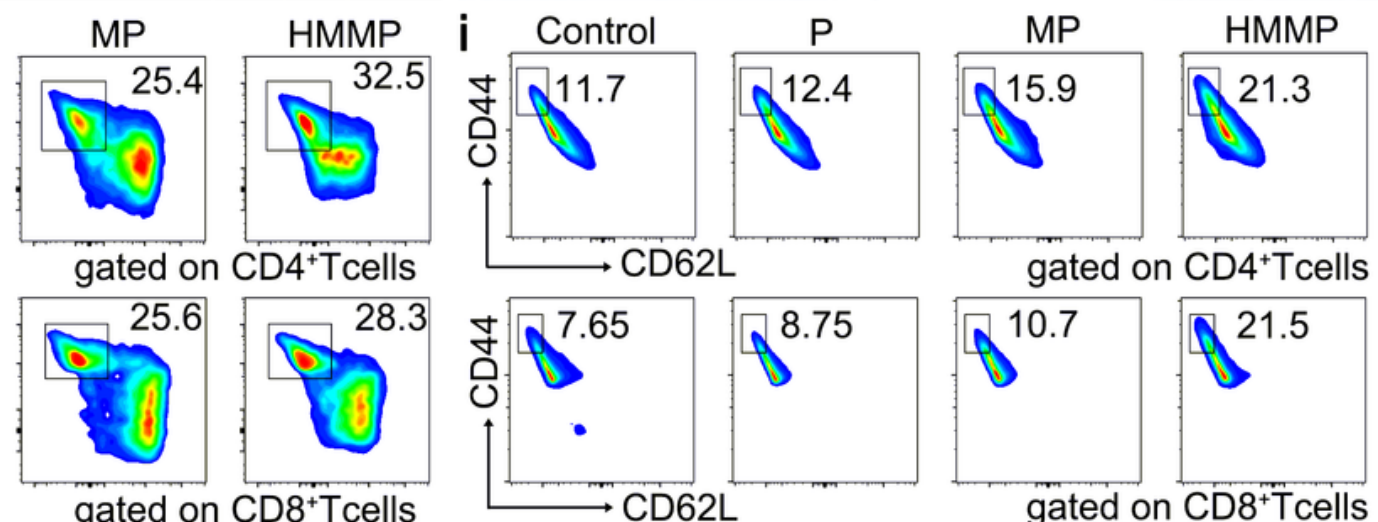

gated on $\mathrm{CD} 4^{+}$Tcells
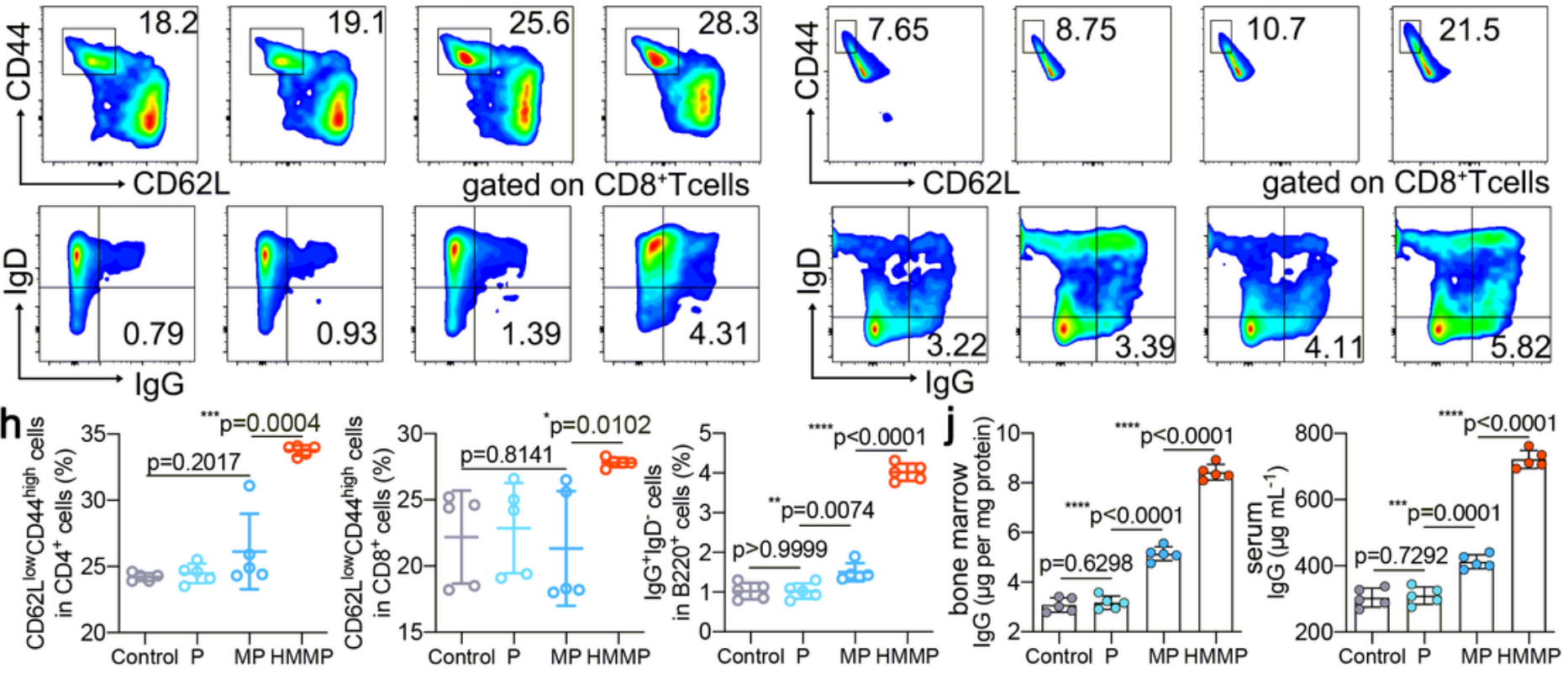

\section{Figure 7}

HMMP in situ vaccination elicits the long-term memory immune response to prevent osteomyelitis recurrence. a, Schematic illustration of the post-surgery personalized vaccine in the recurrent osteomyelitis model. b, Individual and average knee perimeter curves of the re-challenged infected knee. c, Digital photos of the re-challenged infected knee 21 days after the re-implantation and S. aureus injection. $d$, The MRSA CFU counts in the infected bone marrow and extracted implant. e, MRI imaging of 
the reinfected knee. The white circle indicates the reinfected knee. $f$, Gimesa staining images of infected bone marrow. The yellow arrows indicate $S$. aureus. Scale bars, $10 \mu \mathrm{m}$. g,h, Representative flow cytometry plots (g) and quantification (h) of CD4+ memory T cells (CD44highCD62Llow) after gating on CD3+CD4+ cells, CD8+ memory T cells (CD44highCD62Llow) after gating on CD3+CD8+ cells, memory B cells (lgG+lgDlow) after gating on CD45+B220+ cells in the spleens 21 days after the reimplantation and S. aureus injection. $\mathrm{n}=5$ per group. In flow cytometry plots, percentage of cells in the adjacent bound area is indicated. i, Representative flow cytometry plots of CD4+ memory T cells (CD44highCD62Llow) after gating on CD3+CD4+ cells, CD8+ memory T cells (CD44highCD62Llow) after gating on CD3+CD8+ cells, memory B cells (lgG+lgDlow) after gating on CD45+B220+ cells in the bone marrow of re-challenged mice. $j$, IgG concentrations in the bone marrow and serum of re-challenged mice.

\section{Supplementary Files}

This is a list of supplementary files associated with this preprint. Click to download.

- Nat.Nanotechnol.SupplementaryInformation.docx

- Onlinefloatimage1.png 